\title{
Proton decay at one loop
}

\author{
Juan Carlos Helo, ${ }^{1, *}$ Martin Hirsch, ${ }^{2, \dagger}$ and Toshihiko Ota ${ }^{3, \$}$ \\ ${ }^{1}$ Departamento de Física, Facultad de Ciencias, \\ Universidad de La Serena, Avenida Cisternas 1200, La Serena, Chile \\ ${ }^{2}$ AHEP Group, Instituto de Física Corpuscular-CSIC/Universitat de València Edificio \\ de Institutos de Paterna, Apartado 22085, E46071 València, Spain \\ ${ }^{3}$ Departamento de Física Teórica and Instituto de Física Teórica, IFT-UAM/CSIC, \\ Universidad Autónoma de Madrid, Cantoblanco, 28049 Madrid, Spain
}

(Received 10 April 2019; published 20 May 2019)

\begin{abstract}
Proton decay is usually discussed in the context of grand unified theories. However, as is well known, in the standard model effective theory proton decay appears in the form of higher-dimensional nonrenormalizable operators. Here, we study systematically the one-loop decomposition of the $d=6 B+L$ violating operators. We exhaustively list the possible one-loop ultraviolet completions of these operators and discuss that, in general, two distinct classes of models appear. Models in the first class need an additional symmetry in order to avoid tree-level proton decay. These models necessarily contain a neutral particle, which could act as a dark matter candidate. For models in the second class the loop contribution dominates automatically over the tree-level proton decay, without the need for additional symmetries. We also discuss possible phenomenology of two example models, one from each class, and their possible connections to neutrino masses, LHC searches and dark matter.
\end{abstract}

DOI: 10.1103/PhysRevD.99.095021

\section{INTRODUCTION}

While searches for proton decay so far have yielded only lower bounds on the lifetime of various possible decay modes [1-10], future large volume detectors, such as HyperKamiokande [11], DUNE [12] and JUNO [13], or more speculative multimegaton proposals such as TITAND [14,15], MEMPHYS [16] or MICA [17,18] offer a good chance to finally discover this ultrarare process. Although nucleon decay processes are usually discussed in the context of grand unified theories (GUTs), see, e.g., Refs. [19-31], they can arise in many models. For a review on baryon number violation, see for example Ref. [32]. Motivated by the expected improvements in nucleon decay searches, here we study proton decay generated at the one-loop level.

In the standard model baryon and lepton number violation arises at the nonrenormalizable level. At the level of mass dimension five $(d=5)$, there is only one operator, the famous Weinberg operator [33], corresponding to Majorana neutrino masses $(\Delta L=2, \Delta B=0)$. At $d=6$

\footnotetext{
jichelo@userena.cl

mahirsch@ific.uv.es

toshihiko.ota@uam.es
}

Published by the American Physical Society under the terms of the Creative Commons Attribution 4.0 International license. Further distribution of this work must maintain attribution to the author(s) and the published article's title, journal citation, and DOI. Funded by SCOAP. there are already five independent operators, which have $\Delta B=\Delta L=1$ [but $\Delta(B-L)=0$ ] [33-35]. All $d=6$ operators lead to two-body proton decays, such as $p \rightarrow \pi^{0}+e^{+}, p \rightarrow \pi^{+}+\bar{\nu}$ or $p \rightarrow K^{+}+\bar{\nu}$.

GUT models predict proton decay to occur at tree level $[19,20]$. For coefficients of order $\mathcal{O}(1)$, the current experimental bounds then imply a lower limit on the scale of baryon number violation (for $d=6$ operators) of order $\Lambda \sim$ (few) $\mathcal{O}\left(10^{15}\right) \mathrm{GeV}$, which is far out of reach of any foreseeable accelerator experiment. This simple picture changes drastically, if proton decay is induced by higherdimensional operators and/or at loop level. The decay rate for a $k$-body $n$-loop proton (neutron) decay induced by a $d$-dimensional operator can be very roughly estimated to be

$$
\frac{1}{\tau} \sim \frac{\mathcal{C}^{2}}{f[k]}\left(\frac{1}{16 \pi^{2}}\right)^{2 n}\left(\frac{m_{p}}{\Lambda}\right)^{2(d-6)} \frac{m_{p}^{5}}{\Lambda^{4}} .
$$

Here, $f[k] \equiv 4(4 \pi)^{2 k-3}(k-1) !(k-2)$ ! estimates the phase space volume available to the decay products for massless final state particles [36]. The constant $\mathcal{C}$ is the coefficient of the effective interaction that induces the proton decay process, which contains products of couplings that appear in the ultraviolet models given at the scale $\Lambda$. Note that $\mathcal{C}$ can be small compared to one, depending on the model (see below). Obviously, to obtain decay rates within future experimental sensitivities much lower scales $\Lambda$ are needed for $k \gg 2, n \gg 0$ and/or $d \gg 6$. 
Probably for this reason, not many studies on higherdimensional proton decay operators can be found in the literature. For $d=7$ operators see, for example, Refs. [37-39]. For operators with $d=9$ and higher see Refs. [36,40,41]. In particular, Ref. [36] discusses $\Delta L=3$ proton decay from operators up to $d=13$, where current experimental sensitivities correspond to new physics scales $\Lambda \lesssim \mathrm{TeV}$, even for couplings as large as order $\mathcal{O}(1) .{ }^{1}$ The authors of Ref. [43] listed the higher-mass-dimensional $B-L$ violating effective operators in a GUT model and discussed the relations between neutrino masses and the nucleon decays induced by the effective operators.

Even less work has been done so far for loop-induced proton decay. Perhaps the best-known example for it is supersymmetric (SUSY) GUTs, see for example the review [32]. Here, the importance of the loop stems from the fact that the decay amplitude is proportional to $\left(\Lambda_{\mathrm{GUT}} \Lambda_{\mathrm{SUSY}}\right)^{-1}$ instead of $\Lambda_{\text {GUT }}^{-2}$ as (for tree-level contributions) in nonSUSY GUT models.

In this paper, we exhaustively list the possible highenergy completions of the proton decay operators with $d=6$ at the one-loop level. We also calculate group theoretical factors and define the one-loop integrals, which appear in the reproduction of the proton decay operators from their decompositions. From these lists one can immediately estimate the rate of proton decay, once a (proto-)model is specified. For masses of the mediators at the $\mathrm{TeV}$ scale, we find that the couplings $Y$ entering the proton decay rate should be of order $Y<\mathcal{O}\left(10^{-6}\right) .^{2}$ This opens up the possibility that the charged and colored mediator fields live long on the timescale of collider experiments, yielding particular signals at the LHC.

We divide the different models, found in our lists, into two subclasses. Models in the first class require an additional symmetry to avoid tree-level proton decay. It is straightforward to introduce some extra symmetry in these cases, for example a $Z_{2}$ that guarantees that proton decay appears only at the one-loop (and higher) level. In this class of models the lightest loop particle is then necessarily stable and thus can serve as a candidate for the dark matter. In the second class one finds models, in which the loop-induced $d=6$ decay is automatically the leading contribution to proton decay, despite the existence of tree-level decay modes. The reason for this counterintuitive behavior is simply that, for models in the second class, tree-level proton decay appear only at the level of higher-dimensional effective operators.

We then discuss two example models, one from each model class, in more details. In model I, neutrino masses, dark matter and proton decay are all related. Majorana neutrino masses are generated using the scotogenic loop

\footnotetext{
${ }^{1}$ The complete list of high- $d$ operators can easily be obtained with SYM2INT [42].

${ }^{2}$ The coefficient $\mathcal{C}$ in Eq. (1) is $\mathcal{C} \propto Y^{4}$ in one-loop $d=6$ models.
}

[44] and the same $Z_{2}$ that stabilizes the dark matter guarantees that proton decay occurs only at the one-loop level. The colored mediators of proton decay, if at the $\mathrm{TeV}$ scale, can be produced at the LHC and will decay to jets, leptons and the dark matter candidate. These missing energy signals, possibly associated with charged tracks from heavy ionizing particles, are reminiscent of those discussed in the context of SUSY. Thus, one can use different existing searches at the LHC to derive constraints on the model. Also, since the model generates neutrino masses at one loop, one can constrain its parameters using searches for lepton flavor violation, such as $\mu \rightarrow e \gamma$ and others.

In our model II we do not impose any beyond the standard model (SM) symmetry. Thus, there are no stable, heavy particles. Signals for searches at the LHC are therefore different from those discussed for model I. In particular, there are final states with no missing energy involved. For this model, we also show how tree-level proton decay will appear and is suppressed in models in this class. For the particular case of model II, the final state for proton decay is caused by a tree $d=12$ operator and is five-body. The expected partial half-lives for these modes are therefore orders of magnitude larger than those of the one-loop-induced two-body decays.

The rest of this paper is organized as follows. In Sec. II we will discuss the $d=6$ operators and their one-loop decomposition. Section III then presents and discusses our two example models, before we conclude in Sec. IV. Some more technical aspects for the one-loop decomposition are given in the Appendix.

\section{PROTON DECAY OPERATORS AT ONE LOOP}

The effective operators which lead to proton decay were already listed in Refs. [33-35], ${ }^{3}$

$$
\begin{gathered}
\mathcal{O}_{1}=[d u][Q L], \\
\mathcal{O}_{2}=[Q Q][u e], \\
\mathcal{O}_{3}=[Q Q]_{\mathbf{1}}[Q L]_{\mathbf{1}}, \\
\mathcal{O}_{4}=[Q Q]_{\mathbf{3}}[Q L]_{\mathbf{3}}, \\
\mathcal{O}_{5}=[d u][u e],
\end{gathered}
$$

where the subscripts 1 and $\mathbf{3}$ in Eqs. (4) and (5) indicate the electroweak $S U(2)$ representation of the bilinears of the fermions. The contraction of all the indices on the operators is explicitly shown in Appendix.

\footnotetext{
${ }^{3}$ See Refs. [45,46] for the $d=6$ operators for proton decay with a SM singlet fermion (sterile neutrino, also known as righthanded neutrino).
} 


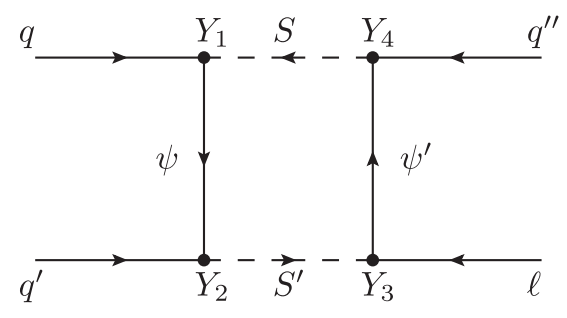

FIG. 1. Box diagram for decomposition of the $d=6$ proton decay operators. The direction of the arrows represents the flow of the particle number (not chirality). We put the lepton field $\ell$ always in the lower right corner $\left(Y_{3}\right)$ in all decompositions, i.e., The $S U(3)$ structure is common in all decompositions.

We are particularly interested in the effective operators which arise from one-loop diagrams. We decompose the effective operators Eqs. (2)-(6) and list the necessary mediators and the interactions. There are two types of topologies for $d=6$ one-loop diagrams: triangle and box. However, the decompositions based on the triangle diagram allow one to have the same effective operator at the tree level. ${ }^{4}$ Therefore, we concentrate on the decompositions with the box diagram. In the decomposition, we distribute the quarks and the lepton of an effective operator to the outer legs of the box diagram shown in Fig. 1 in all possible ways and identify the SM gauge charges of the mediator fields, $\psi, S, \psi^{\prime}$, and $S^{\prime}$. In the current study, we restrict ourselves to the decompositions with scalar and fermion mediators and do not introduce a vector mediator which may require an extension of the SM gauge symmetries and their spontaneous breaking to the SM. In short, we introduce the following Yukawa interactions:

$\mathcal{L}=Y_{1} \overline{q^{c}} \psi^{c} S+Y_{2} \overline{\psi^{c}} q^{\prime} S^{\prime \dagger}+Y_{3} \overline{\psi^{\prime}} \ell S^{\prime}+Y_{4} \overline{q^{\prime \prime c}} \psi^{\prime} S^{\dagger}+$ H.c.,

where $q, q^{\prime}$, and $q^{\prime \prime}$ are the quark fields $(Q, u$, or $d)$, and $\ell$ is the lepton field ( $L$ or $e$ ) in an effective operator. The mediator fields, $\psi$ and $\psi^{\prime}$ for fermions and $S$ and $S^{\prime}$ for scalars, are assigned as shown in Fig. 1. The mass terms for the mediator fields must also be included in the Lagrangian, although they are not explicitly written in Eq. (7). Later we will discuss the phenomenology of mediator fields, assuming that the masses $M_{\psi}, M_{S}, M_{\psi^{\prime}}$, and $M_{S^{\prime}}$ are at the $\mathrm{TeV}$ scale.

The color $S U(3)$ structure of the box diagram Fig. 1 is common in all decompositions, and the possible ways to assign the $S U(3)$ charges to the mediators are listed in

\footnotetext{
${ }^{4}$ Forbidding a Yukawa interaction that mediates a $d=6$ proton decay operator at the tree level by a symmetry and allowing the soft breaking of the symmetry by the mass term of a mediator field, one can induce a triangle diagram solely at the loop level. For more discussions on the realizations, see Ref. [47]. In this study, we do not pursue the possibility of such a setup with a symmetry and its soft breaking.
}

TABLE I. Choices of the $S U(3)$ charges of the mediator fields and the $S U(3)$ coefficients which appear in the reordering of the $S U(3)$ indices to obtain the corresponding effective operator; see Eqs. (8) and (9) and the text.

\begin{tabular}{lccccc}
\hline \hline & \multicolumn{5}{c}{ Mediators } \\
\cline { 2 - 6 } & $\psi$ & $S$ & $\psi^{\prime}$ & $S^{\prime}$ & $S U(3)$ coeff. \\
\hline$\# 1$ & $\mathbf{1}$ & $\overline{\mathbf{3}}$ & $\mathbf{3}$ & $\mathbf{3}$ & -1 \\
$\# 2$ & $\mathbf{3}$ & $\mathbf{1}$ & $\overline{\mathbf{3}}$ & $\overline{\mathbf{3}}$ & 1 \\
$\# 3$ & $\mathbf{3}$ & $\mathbf{8}$ & $\overline{\mathbf{3}}$ & $\overline{\mathbf{3}}$ & $-\frac{8}{3}$ \\
$\# 4$ & $\mathbf{3}$ & $\mathbf{8}$ & $\mathbf{6}$ & $\mathbf{6}$ & 4 \\
$\# 5$ & $\overline{\mathbf{3}}$ & $\mathbf{3}$ & $\mathbf{1}$ & $\mathbf{1}$ & 1 \\
$\# 6$ & $\overline{\mathbf{3}}$ & $\mathbf{3}$ & $\mathbf{8}$ & $\mathbf{8}$ & $-\frac{8}{3}$ \\
$\# 7$ & $\overline{\mathbf{3}}$ & $\overline{\mathbf{6}}$ & $\mathbf{8}$ & $\mathbf{8}$ & -4 \\
$\# 8$ & $\mathbf{6}$ & $\mathbf{3}$ & $\mathbf{8}$ & $\mathbf{8}$ & 4 \\
$\# 9$ & $\overline{\mathbf{6}}$ & $\mathbf{8}$ & $\overline{\mathbf{3}}$ & $\overline{\mathbf{3}}$ & -4 \\
$\# 10$ & $\mathbf{8}$ & $\overline{\mathbf{3}}$ & $\mathbf{3}$ & $\mathbf{3}$ & $\frac{8}{3}$ \\
$\# 11$ & $\mathbf{8}$ & $\overline{\mathbf{3}}$ & $\overline{\mathbf{6}}$ & $\overline{\mathbf{6}}$ & -4 \\
$\# 12$ & $\mathbf{8}$ & $\mathbf{6}$ & $\mathbf{3}$ & $\mathbf{3}$ & 4 \\
\hline \hline
\end{tabular}

Table I. Here we assume that a mediator takes one of $\mathbf{1}, \mathbf{3}, \overline{\mathbf{3}}$, $\mathbf{6}, \overline{\mathbf{6}}$, and $\mathbf{8}$ representations under the $S U(3)$ transformation and do not pursue the possibility of decompositions with a mediator whose representation is higher than 8. In the column "SU(3) coeff.," we also list the coefficients appearing in the calculation, which we call operator projection, to derive the effective operators from the decompositions. In order to obtain the effective operators Eqs. (2)-(6) from the decomposition Eq. (7) where each Yukawa interaction forms a $S U(3)$ singlet, we must rearrange the $S U(3)$ indices as

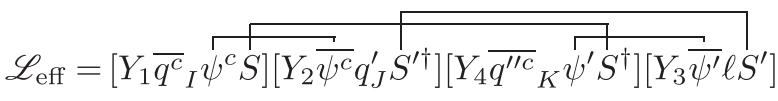

$$
\begin{aligned}
& =Y_{1} Y_{2} Y_{3} Y_{4} \times S U(3) \text { coeff. } \times \epsilon^{I J K}{\overline{q^{c}}}_{I} q_{J}^{\prime} \overline{q^{\prime \prime c}}{ }_{K} \ell \ldots
\end{aligned}
$$

where $I, J$, and $K$ are the $S U(3)$ indices for 3 representations, and $\epsilon^{I J K}$ is the total antisymmetric tensor to form a singlet with three triplets. The part omitted from the second line of Eq. (8), which is expressed as "...," represents all contents other than the Yukawa couplings $\left(Y_{1-4}\right)$, the coefficient $[S U(3)$ coeff.] brought by the rearrangement of the $S U(3)$ indices, and the outer fermion field operators $\left(q, q^{\prime}, q^{\prime \prime}\right.$, and $\ell$ ), such as the propagators of the mediators and matrices with $S U(2)$ indices. We have not specified the quark fields at this stage and rearrange the $S U(3)$ indices by handling them as $\mathbf{3}$ representation field operators in general. Depending on the decomposition with a specific choice of the quark fields, an additional sign can show up in the further rearrangement of the $S U(3)$ indices, which will be taken into account after the full information of the 
TABLE II. Decomposition (protomodels) of the $d=6$ effective operator $\mathcal{O}_{1}=[d u][Q L]$ which consists of $d, u, Q$, and $L$. The protomodels result in the same effective operator $\mathcal{O}_{1}$ but with different coefficients, factors, and signs.

\begin{tabular}{|c|c|c|c|c|c|c|c|}
\hline \multirow[b]{2}{*}{$\mathcal{O}_{1}$ Decom. } & \multicolumn{4}{|c|}{ Mediators $S U(2)_{U(1)}$} & \multirow[b]{2}{*}{$\begin{array}{l}S U(2) \\
\text { coeff. }\end{array}$} & \multirow[b]{2}{*}{$\begin{array}{c}\text { Fierz } \times \text { loop } \\
\text { factors }\end{array}$} & \multirow[b]{2}{*}{$\begin{array}{c}S U(3) \\
\text { sign }\end{array}$} \\
\hline & $\psi$ & $S$ & $\psi^{\prime}$ & $S^{\prime}$ & & & \\
\hline \multirow[t]{4}{*}{$(d u)(Q L)$} & $\mathbf{1}_{\alpha}$ & $\mathbf{1}_{\alpha+\frac{1}{3}}$ & $\mathbf{2}_{\alpha+\frac{1}{6}}$ & $\mathbf{1}_{\alpha+\frac{2}{3}}$ & 1 & $M_{\psi} M_{\psi^{\prime}} I_{4}$ & + \\
\hline & 2 & 2 & 1 & 2 & -1 & & \\
\hline & 2 & 2 & 3 & 2 & -3 & & \\
\hline & 3 & 3 & 2 & 3 & 3 & & \\
\hline \multirow[t]{4}{*}{$(u d)(Q L)$} & $\mathbf{1}_{\alpha}$ & $\mathbf{1}_{\alpha-\frac{2}{3}}$ & $\mathbf{2}_{\alpha-\frac{5}{6}}$ & $\mathbf{1}_{\alpha-\frac{1}{3}}$ & 1 & $M_{\psi} M_{\psi \psi^{\prime}} I_{4}$ & - \\
\hline & 2 & 2 & 1 & 2 & -1 & & \\
\hline & 2 & 2 & 3 & 2 & -3 & & \\
\hline & 3 & 3 & 2 & 3 & 3 & & \\
\hline \multirow[t]{4}{*}{$(d Q)(u L)$} & $\mathbf{1}_{\alpha}$ & $\mathbf{1}_{\alpha+\frac{1}{3}}$ & $\mathbf{1}_{\alpha-\frac{1}{3}}$ & $\mathbf{2}_{\alpha+\frac{1}{6}}$ & -1 & $-\frac{1}{2} J_{4}$ & - \\
\hline & 2 & 2 & 2 & 1 & -1 & & \\
\hline & 2 & 2 & 2 & 3 & 3 & & \\
\hline & 3 & 3 & 3 & 2 & -3 & & \\
\hline \multirow[t]{4}{*}{$(Q d)(u L)$} & $\mathbf{1}_{\alpha}$ & $\mathbf{2}_{\alpha-\frac{1}{6}}$ & $\mathbf{2}_{\alpha-\frac{5}{6}}$ & $\mathbf{1}_{\alpha-\frac{1}{3}}$ & 1 & $\frac{1}{2} J_{4}$ & + \\
\hline & 2 & 1 & 1 & 2 & -1 & & \\
\hline & 2 & 3 & 3 & 2 & -3 & & \\
\hline & 3 & 2 & 2 & 3 & 3 & & \\
\hline \multirow[t]{4}{*}{$(u Q)(d L)$} & $\mathbf{1}_{\alpha}$ & $\mathbf{1}_{\alpha-\frac{2}{3}}$ & $\mathbf{1}_{\alpha-\frac{1}{3}}$ & $\mathbf{2}_{\alpha+\frac{1}{6}}$ & -1 & $-\frac{1}{2} J_{4}$ & + \\
\hline & 2 & 2 & 2 & 1 & -1 & & \\
\hline & 2 & 2 & 2 & 3 & 3 & & \\
\hline & 3 & 3 & 3 & 2 & -3 & & \\
\hline \multirow[t]{4}{*}{$(Q u)(d L)$} & $\mathbf{1}_{\alpha}$ & $\mathbf{2}_{\alpha-\frac{1}{6}}$ & $\mathbf{2}_{\alpha+\frac{1}{6}}$ & $\mathbf{1}_{\alpha+\frac{2}{3}}$ & 1 & $\frac{1}{2} J_{4}$ & - \\
\hline & 2 & 1 & 1 & 2 & -1 & & \\
\hline & 2 & 3 & 3 & 2 & -3 & & \\
\hline & 3 & 2 & 2 & 3 & 3 & & \\
\hline
\end{tabular}

decomposition, with which one can fully specify the ordering of the quark fields. The sign due to the ordering of the quarks will be given in Tables II-V [as " $S U(3)$ sign"]. Note that the $S U(2)$ and the Lorentz indices have not been rearranged at this stage, and the rearrangement of them will bring other coefficients and factors. All the details of the method of decomposition and operator projection are given in Appendix, where we demonstrate the derivation of all the coefficients, signs, and factors, keeping all the indices on the field operators explicitly.

To proceed the operator projection onto the basis operators Eqs. (2)-(6), we must specify the species of the outer fermion fields, determine the position of the quark fields on the box diagram, and identify the $S U(2)$ gauge charges of the mediator fields. In Tables II-V, the ways of decomposition are given in the column "Decom," where the given fermion fields correspond to $\left(q q^{\prime}\right)\left(q^{\prime \prime} \ell\right)$ in Fig. 1 and Eq. (7). The electroweak charges of the mediator fields are listed at the column "Mediators $S U(2)_{U(1)}$ ". We concentrate on $\mathbf{1}, \mathbf{2}$, and $\mathbf{3}$ for the $S U(2)$ representation. Note that
TABLE III. Decomposition of $\mathcal{O}_{2}$, where the ordering of the two quark doublets in the effective operator is determined as $\mathcal{O}_{2} \equiv\left[Q_{1} Q_{2}\right][$ ue $]$.

\begin{tabular}{|c|c|c|c|c|c|c|c|}
\hline \multirow[b]{2}{*}{$\mathcal{O}_{2}$ Decom. } & \multicolumn{4}{|c|}{ Mediators $S U(2)_{U(1)}$} & \multirow[b]{2}{*}{$\begin{array}{l}S U(2) \\
\text { coeff. }\end{array}$} & \multirow[b]{2}{*}{$\begin{array}{c}\text { Fierz } \times \text { loop } \\
\text { factors }\end{array}$} & \multirow[b]{2}{*}{$\begin{array}{c}S U(3) \\
\text { sign }\end{array}$} \\
\hline & $\psi$ & $S$ & $\psi^{\prime}$ & $S^{\prime}$ & & & \\
\hline \multirow[t]{4}{*}{$\left(Q_{1} Q_{2}\right)($ ue $)$} & $\mathbf{1}_{\alpha}$ & $\mathbf{2}_{\alpha-\frac{1}{6}}$ & $\mathbf{2}_{\alpha-\frac{5}{6}}$ & $\mathbf{2}_{\alpha+\frac{1}{6}}$ & 1 & $M_{\psi} M_{\psi^{\prime}} I_{4}$ & + \\
\hline & 2 & 1 & 1 & 1 & 1 & & \\
\hline & 2 & 3 & 3 & 3 & -3 & & \\
\hline & 3 & 2 & 2 & 2 & 3 & & \\
\hline \multirow[t]{4}{*}{$\left(u Q_{2}\right)\left(Q_{1} e\right)$} & $\mathbf{1}_{\alpha}$ & $\mathbf{1}_{\alpha-\frac{2}{3}}$ & $\mathbf{2}_{\alpha-\frac{5}{6}}$ & $\mathbf{2}_{\alpha+\frac{1}{6}}$ & 1 & $\frac{1}{2} J_{4}$ & - \\
\hline & 2 & 2 & 1 & 1 & 1 & & \\
\hline & 2 & 2 & 3 & 3 & -3 & & \\
\hline & 3 & 3 & 2 & 2 & 3 & & \\
\hline \multirow[t]{4}{*}{$\left(Q_{1} u\right)\left(Q_{2} e\right)$} & $\mathbf{1}_{\alpha}$ & $\mathbf{2}_{\alpha-\frac{1}{6}}$ & $\mathbf{1}_{\alpha-\frac{1}{3}}$ & $\mathbf{1}_{\alpha+\frac{2}{3}}$ & 1 & $-\frac{1}{2} J_{4}$ & - \\
\hline & 2 & 1 & 2 & 2 & -1 & & \\
\hline & 2 & 3 & 2 & 2 & -3 & & \\
\hline & 3 & 2 & 3 & 3 & 3 & & \\
\hline
\end{tabular}

the sign that comes up in the rearrangement of the $S U(3)$ indices are also given in the column of the $S U(3)$ sign in Tables II-V, which cannot be included in Table I because they depend on the ordering of the quark fields in a decomposition. We also list the factors and coefficients which come up in the process of the operator projection after Eq. (8): the coefficients and signs from the rearrangement of the $S U(2)$ indices [SU(2) coeff.], the factors and signs from the Fierz transformations (rearrangement of the Lorentz indices), and the loop integral factors ("Fierz $\times$ loop factors"). The functions $I_{4}$ and $J_{4}$ for the loop integrals of the box diagrams are defined in the Appendix. In short, once the decomposition (protomodel) is specified (one from Table I and one from Tables II-V are chosen), the coefficient of the effective operator is given as

$$
\begin{aligned}
\mathcal{L}_{\text {eff }}= & S U(3) \text { coeff } \times S U(3) \text { sign } \times S U(2) \text { coeff } \\
& \times \text { Fierz factor } \times \text { loop factor } \times Y_{1} Y_{2} Y_{3} Y_{4} \\
& \times \text { effective op }(\mathrm{s}) \mathcal{O} \text { in Eqs. }(2)-(6),
\end{aligned}
$$

with which, and also with the help of the nucleon matrix elements calculated from lattice [48-51] and chiral perturbation theory [52-54], one can directly calculate the rates of proton decay. The notations and the derivations of the coefficients, factors, and signs are given in the Appendix.

Here we show an example to demonstrate how to use the information of the tables. A famous dimension-five contribution to proton decay in SUSY-GUT models is found by taking \#1 from Table I and the decomposition of the seventh row in Table IV with $\alpha=0$ for $U(1)$ hypercharge. The mediators are identified with the SUSY particles as 
TABLE IV. Decompositions of the effective operators with three $Q$ s and a $L$. Each model results in a different combination of $\mathcal{O}_{3}$ and $\mathcal{O}_{4}$. Note that the ordering of the three $Q \mathrm{~s}$ in the effective operators are fixed as $\mathcal{O}_{3 / 4} \equiv\left[Q_{1} Q_{2}\right]_{1 / 3}\left[Q_{3} L\right]_{1 / 3}$.

\begin{tabular}{|c|c|c|c|c|c|c|c|}
\hline \multirow[b]{2}{*}{$\mathcal{O}_{3,4}$ Decom. } & \multicolumn{4}{|c|}{ Mediators $S U(2)_{U(1)}$} & \multirow[b]{2}{*}{$S U(2)$ coeff. } & \multirow[b]{2}{*}{ Fierz $\times$ loop factors } & \multirow[b]{2}{*}{$S U(3)$ sign } \\
\hline & $\psi$ & $S$ & $\psi^{\prime}$ & $S^{\prime}$ & & & \\
\hline \multirow[t]{8}{*}{$\left(Q_{1} Q_{2}\right)\left(Q_{3} L\right)$} & $\mathbf{1}_{\alpha}$ & $\mathbf{2}_{\alpha-\frac{1}{6}}$ & $\mathbf{1}_{\alpha-\frac{1}{3}}$ & $\mathbf{2}_{\alpha+\frac{1}{6}}$ & $-\frac{1}{2} \mathcal{O}_{3}+\frac{1}{2} \mathcal{O}_{4}$ & $M_{\psi} M_{\psi^{\prime}} I_{4}$ & + \\
\hline & 1 & $2^{6}$ & $3^{3}$ & 2 & $-\frac{3}{2} \mathcal{O}_{3}-\frac{1}{2} \mathcal{O}_{4}$ & & \\
\hline & 2 & 1 & 2 & 1 & $\mathrm{O}_{3}{ }^{2}$ & & \\
\hline & 2 & 1 & 2 & 3 & $\mathcal{O}_{4}$ & & \\
\hline & 2 & 3 & 2 & 1 & $-\mathcal{O}_{4}$ & & \\
\hline & 2 & 3 & 2 & 3 & $-3 \mathcal{O}_{3}+2 \mathcal{O}_{4}$ & & \\
\hline & 3 & 2 & 1 & 2 & $-\frac{3}{2} \mathcal{O}_{3}-\frac{1}{2} \mathcal{O}_{4}$ & & \\
\hline & 3 & 2 & 3 & 2 & $-\frac{9}{2} \mathcal{O}_{3}+\frac{1}{2} \mathcal{O}_{4}$ & & \\
\hline
\end{tabular}

$$
\begin{aligned}
\psi(\mathbf{1}, \mathbf{3})_{0} & =\tilde{W}, & & S(\overline{\mathbf{3}}, \mathbf{2})_{-1 / 6}=\tilde{Q}^{*}, \\
\psi^{\prime}(\mathbf{3}, \mathbf{1})_{-1 / 3} & =\tilde{h}_{c}, & & S^{\prime}(\mathbf{3}, \mathbf{2})_{+1 / 6}=\tilde{Q},
\end{aligned}
$$

where $\tilde{W}$ is the wino, $\tilde{h}_{c}$ is the colored higgsino, and $\tilde{Q}$ is the squark doublet. Using the information listed in the tables, we can reproduce the coefficient of the effective operator (9) as

$$
\begin{aligned}
\mathcal{L}_{\text {eff }}= & \overbrace{(-1)}^{S U(3) \text { coeff }} \times \overbrace{(+)}^{S U(3) \text { sign }} \times \overbrace{\left[-\frac{3}{2} \mathcal{O}_{3}-\frac{1}{2} \mathcal{O}_{4}\right]}^{S U(2) \text { coeff and } \mathcal{O}} \\
& \times \overbrace{M_{\psi} M_{\psi^{\prime}} I_{4}}^{\text {Fierz×loop factors }} \times Y_{1} Y_{2} Y_{3} Y_{4} \\
= & \frac{1}{2} Y_{1} Y_{2} Y_{3} Y_{4} M_{\tilde{W}} M_{\tilde{h}_{c}} I_{4}\left[3 \mathcal{O}_{3}+\mathcal{O}_{4}\right] .
\end{aligned}
$$

Note that $Y_{1}$ and $Y_{2}$ are given by the gauge coupling in $S U(5)$ SUSY-GUT models. The coupling $Y_{3}$ is identified with the coupling for the Yukawa interaction of $\mathbf{1 0} \cdot \overline{\mathbf{5}} \cdot H(\overline{\mathbf{5}})$, and $Y_{4}$ is that for $\mathbf{1 0} \cdot \mathbf{1 0} \cdot H(\mathbf{5})$, where $\mathbf{1 0}$ and $\overline{\mathbf{5}}$ are the matter superfields and $H(\overline{\mathbf{5}})$ and $H(\mathbf{5})$ are the Higgs superfields. Taking the decomposition of the first row in Table IV, one can find the same diagram but with a bino $\tilde{B}(\mathbf{1}, \mathbf{1})_{0}$ instead of the wino $\tilde{W}$.

\section{MODELS AND PHENOMENOLOGY}

In this section we discuss the phenomenology of the different one-loop models presented above. We will start with a brief discussion of the different model classes and an overview of commonalities that all these one-loop models share. We then discuss two example models in some more detail.

\section{A. General discussion}

Our results listed in Tables I-V summarize the possible particle content that allows us to construct models with oneloop-induced proton decay, we call this the "protomodels." However, not all allowed choices of quantum numbers will automatically result in models in which the one-loop

\begin{tabular}{|c|c|c|c|c|c|c|}
\hline \multirow[b]{2}{*}{$\mathcal{O}_{5}$ Decom. } & \multicolumn{4}{|c|}{ Mediators $S U(2)_{U(1)}$} & \multirow[b]{2}{*}{$S U(2)$ coeff. } & \multirow[b]{2}{*}{ Fierz $\times$ loop factors $\times S U(3)$ sign } \\
\hline & $\psi$ & $S$ & $\psi^{\prime}$ & $S^{\prime}$ & & \\
\hline \multirow[t]{3}{*}{$\left(d u_{1}\right)\left(u_{2} e\right)$} & $\mathbf{1}_{\alpha}$ & $\mathbf{1}_{\alpha+\frac{1}{3}}$ & $\mathbf{1}_{\alpha-\frac{1}{3}}$ & $\mathbf{1}_{\alpha+\frac{2}{3}}$ & 1 & $M_{\psi} M_{\psi^{\prime}} I_{4} \mathcal{O}_{5}$ \\
\hline & 2 & 2 & 2 & 2 & 2 & \\
\hline & 3 & 3 & 3 & 3 & 3 & \\
\hline \multirow[t]{3}{*}{$\left(u_{1} d\right)\left(u_{2} e\right)$} & $\mathbf{1}_{\alpha}$ & $\mathbf{1}_{\alpha-\frac{2}{3}}$ & $\mathbf{1}_{\alpha-\frac{4}{3}}$ & $\mathbf{1}_{\alpha-\frac{1}{3}}$ & 1 & $-M_{\psi} M_{\psi^{\prime}} I_{4} \mathcal{O}_{5}$ \\
\hline & 2 & 2 & 2 & 2 & 2 & \\
\hline & 3 & 3 & 3 & 3 & 3 & \\
\hline \multirow[t]{3}{*}{$\left(u_{2} u_{1}\right)(d e)$} & $\mathbf{1}_{\alpha}$ & $\mathbf{1}_{\alpha-\frac{2}{3}}$ & $\mathbf{1}_{\alpha-\frac{1}{3}}$ & $\mathbf{1}_{\alpha+\frac{2}{3}}$ & 1 & $M_{\psi} M_{\psi^{\prime}} I_{4}\left[\mathcal{O}_{5}-\mathcal{O}_{5}^{\prime}\right]$ \\
\hline & 2 & $2^{3}$ & $2^{3}$ & 2 & 2 & \\
\hline & 3 & 3 & 3 & 3 & 3 & \\
\hline
\end{tabular}
contribution to proton decay will be the dominant one. To see this in a simple example, consider decomposition \#1

TABLE V. Decompositions of $\mathcal{O}_{5}$. Here the ordering of the two $u$ 's in the basis operators is determined as $\mathcal{O}_{5} \equiv\left[d u_{1}\right]\left[u_{2} e\right]$ and $\mathcal{O}_{5}^{\prime} \equiv\left[d u_{2}\right]\left[u_{1} e\right]$. 
from Table I and the decomposition of the third row in Table IV with $\alpha=1 / 2$. In this case $S$ is identified with $S(\overline{\mathbf{3}}, \mathbf{1})_{1 / 3}$. The quantum numbers of this scalar allow us to write down the following two interactions with standard model fermions: $Q Q S^{\dagger}$ and $Q L S$. The product of these interactions, after integrating out $S$, generate $\mathcal{O}_{3}$ at tree level. ${ }^{5}$ Thus, unless there is a strong hierarchy between the different Yukawa interactions, one expects that the treelevel contribution dominates the decay rate. One can eliminate such an unwanted hierarchy in couplings using additional symmetries. The simplest possibility is to just assign the particles running in the loop to be odd under a $Z_{2}$, while all the standard model particles are even. We classify models, which need such an additional symmetry to avoid unwanted tree-level proton decay, as class-I models. We discuss one example model from this class in Sec. III B.

In addition, there exist choices of quantum numbers, for which tree-level two-body decays are not allowed, but higher multiplicity final states are generated at tree-level together with the one-loop diagrams. For example, if $S$ and $S^{\prime}$ are chosen to be $S(\mathbf{3}, \mathbf{2})_{1 / 6}$ and $S^{\prime}(\mathbf{1}, \mathbf{2})_{1 / 2}$, which have the same charges as a scalar leptoquark and the SM Higgs field, the corresponding models will produce three-body proton decays, such as $p \rightarrow \pi^{+} \pi^{+} e^{-}$via an effective $d=9$ operator. In such cases, one expects in general that the $d=6$ one-loop operator dominates over the $d=9$ treelevel operator for typical scales $\Lambda \gtrsim 1 \mathrm{TeV}$, see Eq. (1). However, if there is some hierarchy in the Yukawa couplings, $\left(Y_{3} Y_{4}\right) \ll\left(Y_{1} Y_{2}\right)$, one can arrange the threebody decays to dominate over the two-body ones and one needs again a symmetry to assure that the loop dominates over the tree-level contribution. Our first example model is exactly of this type, see Sec. III B.

Finally, there are choices, where the particle content of the one-loop model is such that tree-level proton decay can occur only at $d=12$ and higher (usually leading to proton decay with five-body final states). In these cases the oneloop $d=6$ decay will win over the tree-level decays for all practical choices of model parameters. We consider such models interesting and define these models as class-II models, since no symmetry is required to make the oneloop $d=6$ decays dominant. We will discuss one concrete example model in Sec. III C.

Obviously, the main difference between models in class I and class II is that in class I the lightest particle will be absolutely stable. This opens up the possibility to connect proton decay to dark matter, but requires an electrically neutral particle in the loop. We will come back to a more detailed discussion of this point in Sec. III B.

${ }^{5} S(\overline{\mathbf{3}}, \mathbf{1})_{1 / 3}$ is not the only choice that will lead to tree-level proton decay. The same argument applies to $S(\overline{\mathbf{3}}, \mathbf{3})_{1 / 3}$ and $S(\overline{\mathbf{3}}, \mathbf{1})_{4 / 3}$.
Let us now turn to a rough estimate of the proton decay half-life. Using results from lattice QCD calculations [48-51] and chiral perturbation theory [52-54], the twobody proton decay half-life can be calculated as ${ }^{6}$

$$
\begin{aligned}
\tau & \simeq \frac{1}{\frac{m_{p}}{32 \pi}\left[1-\frac{m_{\text {meson }}^{2}}{m_{p}^{2}}\right]^{2}\left|W \frac{Y_{1} Y_{2} Y_{3} Y_{4}}{16 \pi^{2} \cdot 6 \cdot M^{2}}\right|^{2}} \\
& \sim 10^{34}(\mathrm{yr})\left[\frac{M}{1 \mathrm{TeV}}\right]^{4}\left[\frac{3 \times 10^{-6}}{\bar{Y}}\right]^{8} .
\end{aligned}
$$

Note that half-life estimates for different operators, $\mathcal{O}_{1}-\mathcal{O}_{5}$, differ slightly due to the different possible final states. For the numerical estimate we use the charged pion mass for the mass $m_{\text {meson }}$ of the daughter meson. $W$ is the corresponding hadronic matrix element, $W \equiv\langle\operatorname{meson}|(q q) q| p\rangle=-0.181$ $\left[\mathrm{GeV}^{2}\right]$; the numerical value has recently been calculated in Ref. [49]. The factor $1 / 6$ in the first equation above is due to the loop integral $I_{4}$, in the limit of equal masses. We have defined the mean coupling $\bar{Y}=\left(Y_{1} Y_{2} Y_{3} Y_{4}\right)^{1 / 4}$, since proton decay is sensitive only to this product, and used a mass scale of $1 \mathrm{TeV}$, since we are interested in possible LHC phenomenology of these one-loop models.

With couplings of the order of Eq. (12), the particles in the one-loop diagrams can be rather long-lived. Depending on the choices of parameters, i.e., Yukawa couplings and mass hierarchies of the new particles, decay lengths can vary from unmeasurably short to many meters. The collider phenomenology of long-lived particles has recently attracted a lot of attention in the literature, see for example Refs. [55-62]. There are also plans for several future experiments, dedicated to the search for ultralong-lived particles, see for example Refs. [63-67]. For the current status of searches for longlived particles at the LHC, see Refs. [68-85]. We will come back to a more detailed discussion of the LHC phenomenology of our one-loop models in Secs. III B and III C.

\section{B. Model I}

Here we discuss one example model, corresponding to the choices \#5 in Table I for color, the second row in Table II for the decomposition of $\mathcal{O}_{1}$, and the parameter $\alpha$ for the electroweak $U(1)$ hypercharge to $-1 / 6$. The SM charges of the mediator fields are then determined as

$$
\psi(\overline{\mathbf{3}}, \mathbf{2})_{-1 / 6}, \quad S(\mathbf{3}, \mathbf{2})_{+1 / 6}, \quad \psi^{\prime}(\mathbf{1}, \mathbf{1})_{0}, \quad S^{\prime}(\mathbf{1}, \mathbf{2})_{+1 / 2} .
$$

Note that the scalar mediator field $S^{\prime}$ has the same charges as the SM Higgs field $H$. The one-loop diagram for proton decay is shown in Fig. 2.

\footnotetext{
${ }^{6}$ The simple estimate (1) would give $\bar{Y}$ roughly a factor 2.5 smaller.
} 
As discussed above, the model allows for a $d=9$ three-body decay of the proton, unless an additional symmetry is introduced. This can be seen easily as follows: Cutting the diagram in the scalar lines, one obtains the effective operator $d_{R} u_{R} S S^{\prime \dagger}$. Including the decays of the scalars produces then a $\Delta(B-L)=2 d=9$ operator: $\left(d_{R} u_{R}\right)\left(\bar{L} d_{R}\right)\left(\overline{u_{R}} Q\right)$. It is easy to forbid this operator, simply postulating $B-L$ conservation, since the original $d=6$ operator has $\Delta(B-L)=0$. More interesting phenomenologically, however, is to assign a new $Z_{2}$ to the model, under which all loop particles are odd, while the SM fermions are even. In this case, unless the $Z_{2}$ is spontaneously broken, the lightest of the particles in the loop is absolutely stable and can be therefore a candidate for the dark matter. In the following, we will discuss this variant of the model.

Combining the information listed in Tables I and II, one can find the coefficient of the effective interaction of proton decay processes as

$$
\mathcal{C}_{1}=-M_{\psi} M_{\psi^{\prime}} I_{4} Y_{1} Y_{2} Y_{3} Y_{4}
$$

with which the effective Lagrangian (9) is given as $\mathcal{L}_{\text {eff }} \equiv \mathcal{C}_{1} \mathcal{O}_{1}$. The effective operator $\mathcal{O}_{1}$ causes decay of a proton in two modes, and the rates are calculated with the coefficient Eq. (14) as ${ }^{7}$

$\Gamma\left(p \rightarrow \pi^{+} \bar{\nu}_{e} / \pi^{0} e^{+}\right)=\frac{m_{p}}{32 \pi}\left[1-\frac{m_{\pi^{+/ 0}}^{2}}{m_{p}^{2}}\right]^{2}\left|W_{0} \mathcal{C}_{1}\right|^{2}$,

where the hadronic matrix elements $W_{0}$ are found in Ref. [49]: $W_{0}=-0.186(-0.131)\left(\mathrm{GeV}^{2}\right)$ for the $\pi^{+}\left(\pi^{0}\right)$ mode. All decompositions in Table II, which result in the operator $\mathcal{O}_{1}$, predict roughly the same size of the rates for both decay modes. Therefore, if it turns out that the rates of the two modes are very different, models based on $\mathcal{O}_{1}$ will be disfavored. As we have already seen in Eq. (12), the mean of the couplings $\bar{Y}=\left(Y_{1} Y_{2} Y_{3} Y_{4}\right)^{1 / 4}$ should be order few $\mathcal{O}\left(10^{-6}\right)$ for masses accessible at the LHC.

The interaction $Y_{3} \overline{\psi^{\prime}}(\mathbf{1}, \mathbf{1})_{0} L S^{\prime}(\mathbf{1}, \mathbf{2})_{+1 / 2}$ in Fig. 2 can be identified with the corresponding interaction that appears in the scotogenic model [44], since $\psi^{\prime}(\mathbf{1}, \mathbf{1})_{0}$ can be interpreted as a $\nu_{R}$. Note that model I is not the only decomposition that contains such an interaction. Requiring the fields $\left(\psi^{\prime}\right.$ and $\left.S^{\prime}\right)$ relevant for the radiative neutrino mass generation to be color singlets, we have only one choice left for the assignment of the color charges, which is \#5 in Table I. Assuming the $\psi^{\prime}$ to be a singlet under the electroweak $S U(2)$ as in the original scotogenic model, we have the choices of Nos. 2, 6, 9, 14, 17, 22 in Table II for

\footnotetext{
${ }^{7}$ In this estimate we do not take into account the effect of the renormalization group running $[35,45]$ of the operators and use the coefficient at the scale of the proton mass. This is sufficient for our rough estimates.
}

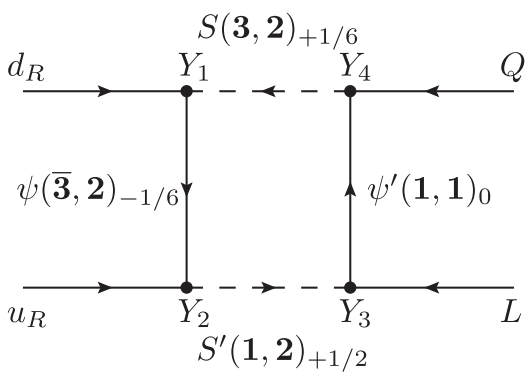

FIG. 2. An example, model I, for a one-loop decomposition of the proton decay operator $\mathcal{O}_{1}$. This model relates proton decay to neutrino masses, the dark matter, and possibly a long-lived colored particle at the LHC; compare with Fig. 3.

$\mathcal{O}_{1}$ and Nos. 1 and 7 in Table IV for $\mathcal{O}_{3,4}$. In total, we have eight possibilities for loop-induced proton decays which can accommodate neutrino masses and dark matter with the scotogenic-type realization. ${ }^{8}$

The phenomenology of the scotogenic model has been studied in many papers, see for example Refs. [86-98]. We will therefore only briefly summarize the most important aspects of its phenomenology here and comment on the differences between our model I and the original scotogenic model.

To generate a Majorana mass term for neutrinos from the one-loop diagram (upper left in Fig. 3) one introduces a scalar quartic interaction between the SM Higgs and the new scalar [44]

$$
\mathcal{L} \supset \lambda_{5}\left(S^{\prime \dagger} H\right)\left(S^{\prime \dagger} H\right)+\text { H.c. }
$$

The flavor structure of the Majorana mass term for neutrinos in the scotogenic model can be expressed as

$$
\left(m_{\nu}\right)^{\alpha \beta}=\sum_{i}\left(Y_{3}^{\top}\right)^{\alpha}{ }_{i} \Lambda_{i}\left(Y_{3}\right)_{i}{ }^{\beta},
$$

which shows that at least two $\psi^{\prime}$ are necessary to reproduce the two mass squared differences measured by neutrino oscillation data. One can find the loop integral $\Lambda_{i}$ in Ref. [44], which is

$$
\Lambda_{i} \equiv \frac{M_{\psi_{i}^{\prime}}}{16 \pi^{2}}\left[f\left(M_{\psi_{i}^{\prime}}^{2} / M_{\mathrm{Re} S^{\prime 0}}^{2}\right)-f\left(M_{\psi_{i}^{\prime}}^{2} / M_{\mathrm{Im} S^{\prime 0}}^{2}\right)\right],
$$

where the function $f(x)$ is defined as $f(x)=$ $-\ln t /(1-x)$. The splitting between the mass $M_{\mathrm{ReS}^{\prime}}$ of the real part of the neutral component of $S^{\prime}$ and $M_{\mathrm{Im} S^{\prime}}$ of

\footnotetext{
${ }^{8}$ The scotogenic-type diagram for neutrino masses can be drawn with the Majorana fermion $\left(\psi^{\prime}\right)$ in the adjoint representations under the SM gauge symmetries. If we relax the requirements to include the adjoint representations, we have more possibilities: Nos. 6-8 in Table I for color, and Nos. 3, 7, 12, 15, 20, and 23 in Table II for $\mathcal{O}_{1}$ and Nos. 2 and 8 in Table IV for $\mathcal{O}_{3,4}$.
} 

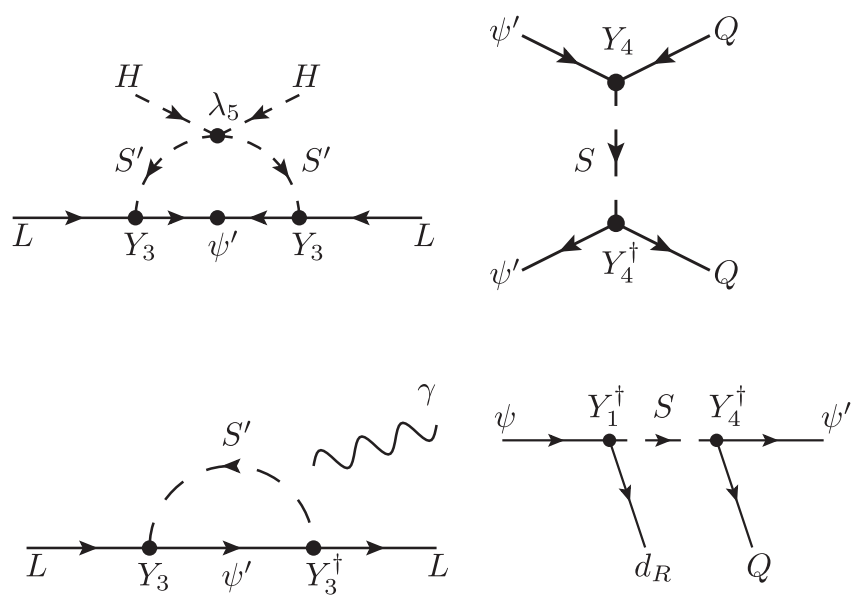

FIG. 3. Phenomenology of model I: Majorana masses for neutrinos (upper left), example of a dark matter (DM) annihilation process for the freeze-out scenario (upper right), a charged lepton flavor violating process (lower left), and a decay chain of the colored mediator field $\psi$ at the LHC (lower right).

the imaginary part is given by the scalar mixing term (16) as $M_{\operatorname{ReS}^{\prime 0}}^{2}-M_{\mathrm{Im} S^{\prime 0}}^{2}=2 \lambda_{5}\left\langle H^{0}\right\rangle^{2}$. It is clear from Eq. (18) and also the diagram in Fig. 3 that the loop integral vanishes in the limit where the mass splitting, which is proportional to the scalar mixing, goes to zero. In short, the size of the neutrino masses is controlled by the scalar mixing coupling $\lambda_{5}$, the Yukawa interaction $Y_{3}$, and the masses of the mediators. Here we are interested in the phenomenology of the mediators with masses of the $\mathrm{TeV}$ scale. Setting the mediator masses to roughly a $\mathrm{TeV}$, one finds that either $Y_{3}$ should be small, say of the order of $\mathcal{O}\left(10^{-5}\right)$ for $\lambda_{5}$ order $\mathcal{O}(1)$, or $\lambda_{5}$ should be order $\mathcal{O}\left(10^{-10}-10^{-8}\right)$ for Yukawas order $0.1-1$ to obtain $m_{\nu} \sim \mathcal{O}(0.1) \mathrm{eV}$.

The $Y_{3}$ interaction also mediates charged lepton flavor violating (CLFV) processes. Studies with a parameter scan, e.g., Refs. [94,96], conclude that the $\ell_{\alpha} \rightarrow \ell_{\beta} \gamma$ processes currently places the most stringent constraints on the model parameters in a wide area of the parameter space. A general formula for the rate of this CLFV process has been presented in Ref. [99]. It can be written as

$\Gamma\left(\ell_{\alpha} \rightarrow \ell_{\beta} \gamma\right)=\frac{e^{2} m_{\ell_{\alpha}}^{5}}{16 \pi}\left|\sum_{i}\left(Y_{3}^{\dagger}\right)_{\beta}{ }^{i}\left(Y_{3}\right)_{i}{ }^{\alpha}\left[-\bar{c}+\frac{3}{2} \bar{d}\right]_{i}\right|^{2}$

The loop integral factor in Eq. (19) is given as

$$
\left[-\bar{c}+\frac{3}{2} \bar{d}\right]_{i}=\frac{\mathrm{i}}{16 \pi^{2}} \frac{1}{M_{S^{\prime}}^{2}}\left[\frac{2 t_{i}^{2}+5 t_{i}-1}{12\left(t_{i}-1\right)^{3}}-\frac{t_{i}^{2} \ln t_{i}}{2\left(t_{i}-1\right)^{4}}\right],
$$

with $t_{i} \equiv M_{\psi_{i}^{\prime}}^{2} / M_{S^{\prime+}}^{2}$. The nonobservation of the $\mu \rightarrow e \gamma$ process [100] suggests $\left(Y_{3}\right)_{i}{ }^{\alpha \in\{e, \mu\}} \lesssim \mathcal{O}(0.01-1)$ for mediators with masses of $\mathcal{O}(1) \mathrm{TeV}$ [96]. For future prospects of the experimental bounds to the CLFV processes are summarized in, e.g., Ref. [101].

We now turn to a brief discussion of dark matter. There are two possible candidates in model I. The scalar $S^{\prime}(\mathbf{1}, \mathbf{2})_{+1 / 2}$ can be identified with the inert doublet, discussed many times in the literature. For inert doublet DM see, for example, Refs. [102,103]. The second candidate is the neutral fermion. For a detailed study of singlet fermion DM in the scotogenic model see, for example, Refs. [96,98].

Suppose the lightest Majorana fermion $\psi_{1}^{\prime}$ is the DM field and was thermally produced and frozen out in the early Universe. In our model, there are two pair-annihilation modes for $\psi_{1}^{\prime}$, which are $\psi_{1}^{\prime} \psi_{1}^{\prime} \rightarrow Q \bar{Q}$ and $L \bar{L}$. The $L \bar{L}$ mode, mediated by the $Y_{3}$ interaction, is the only mode in the original scotogenic model. For this diagram to be efficient enough, $Y_{3}$ should be large, which produces a mild tension between upper limits from CLFV and the minimal $Y_{3}$ required to reproduce the correct relic density [96]. ${ }^{9}$ However, the model we are discussing here also has the interaction $Y_{4}$ of the DM field $\psi^{\prime}$ with $Q$ and can annihilate through the $\psi^{\prime} \psi^{\prime} \rightarrow Q \bar{Q}$ channel, see Fig. 3. The cross section for this pair-annihilation process can be roughly estimated as

$$
\begin{aligned}
\sum_{q=u, d}\left\langle\sigma\left(\psi^{\prime} \psi^{\prime} \rightarrow q \bar{q}\right) v\right\rangle & \sim 2 \times \pi\left[\frac{\left|Y_{4}\right|^{2}}{4 \pi}\right]^{2} \frac{1}{M^{2}} \times \frac{T}{M} \\
& =2 \times 10^{-26}\left[\mathrm{~cm}^{3} / \mathrm{s}\right]\left[\frac{Y_{4}}{1.0}\right]^{4}\left[\frac{\mathrm{TeV}}{M}\right]^{2},
\end{aligned}
$$

where $T$ is the freeze-out temperature. The suppression factor $T / M \sim 1 / 20$ comes from the fact that the annihilation amplitude is $p$-wave, since the initial state consists of two Majorana fermions (cf., e.g., Ref. [104]). Note that, cross sections of order $2 \times 10^{-26}\left(\mathrm{~cm}^{3} / \mathrm{s}\right)$ will reproduce the correct relic density. Again, as in the case of $Y_{3}$, much smaller values of this coupling would be sufficient, if $S(\mathbf{3}, \mathbf{2})_{+1 / 6}$ is not much heavier than $\psi^{\prime}$, such that coannihilation effects become important.

Finally, we will discuss the LHC phenomenology of model I. We will concentrate on the colored states $S(\mathbf{3}, \mathbf{2})_{+1 / 6}$ and $\psi(\overline{\mathbf{3}}, \mathbf{2})_{-1 / 6}$. Let us first consider $S$. This scalar will decay to a two-body final state of jets $(j)$ plus missing energy $(E)$. The decay will be prompt, unless $Y_{4}$ is tiny, say $Y_{4} \ll 10^{-7}$. Thus, limits from standard SUSY searches apply. For example, CMS has searched for scalar

\footnotetext{
${ }^{9}$ It is possible to solve this problem of the overabundance of DM field in scotogenic models in regions of parameter space where coannihilation processes are sizeable, see, for example, Refs. [96,98].
} 
quarks decaying promptly to jets plus missing energy [105]. The limits for one generation of squarks reach up to $1 \mathrm{TeV}$ for neutralino mass of $m_{\tilde{\chi}^{0}} \sim 100 \mathrm{GeV}$ and weaken to roughly $600 \mathrm{GeV}$ for $m_{\tilde{\chi}^{0}} \sim 400 \mathrm{GeV}$ [105]. Similar numbers can be found in ATLAS searches, for example, [106].

The possible decay chains for the colored fermion $\psi^{c}(\mathbf{3}, \mathbf{2})_{+1 / 6}$ lead to final states of either $j j E^{\epsilon}$ or $j l^{ \pm} \mathscr{E}$. Thus, even though $\psi$ resembles a vectorlike quark (VLQ) from its quantum numbers, standard VLQ searches do not apply to this state. (For a summary of CMS searches for VLQs see, for example, Ref. [107].) On the other hand, pair production of $\psi$ will lead to final states that resemble again those for SUSY searches for squarks and gluinos. However, which of the LHC searches can be used to constrain the $\psi$ depends on whether its decays are prompt or not. This in turn depends on the mass hierarchy of the particles in the loop, see Fig. 3. If the mass $M_{\psi}$ of $\psi$ is larger than either of the masses of the scalars $S(\mathbf{3}, \mathbf{2})_{+1 / 6}$ or $S^{\prime}(\mathbf{1}, \mathbf{2})_{+1 / 2}$, the decays of $\psi$ are two body and likely prompt, unless again the corresponding Yukawa is considerably smaller than the estimate for $\bar{Y}$ of $\mathcal{O}\left(10^{-6}\right)$ discussed above from proton decay sensitivities. ATLAS gives lower limits on the gluino mass in simplified SUSY models of order $m_{\tilde{g}} \gtrsim 2 \mathrm{TeV}$ [106]. However, the limits on $\psi$ will be weaker, since (a) the cross section for a color triplet is smaller than for the gluino (octet) and (b) the $\psi$ can also decay to $j l^{ \pm} E^{t}$ with an unknown branching ratio, so this zero-lepton search [106] does not always directly apply. Masses $M_{\psi}$ below $1 \mathrm{TeV}$ will, however, always be excluded since ATLAS leptoquark searches [108] can be combined with the SUSY search [106], as long as the decays of $\psi$ are prompt.

Assume now that $M_{\psi}$ is smaller than the mass $M_{S}$ of the scalar $S(\mathbf{3}, \mathbf{2})_{+1 / 6}$. The three-body decay rate of $\psi$ to $j j E$ can then be estimated as

$$
\Gamma\left(\psi(\overline{\mathbf{3}}, \mathbf{2})_{-1 / 6} \rightarrow j j \mathscr{E}\right) \simeq \frac{\left|Y_{1} Y_{4}\right|^{2}}{512 \pi^{3}}\left[\frac{M_{\psi}}{M_{S}}\right]^{4} M_{\psi}
$$

Note that the rate for the three-body final state $j l^{ \pm} \not E$ is given by the same expression, simply replacing $\left|Y_{1} Y_{4}\right|$ with $\left|Y_{2} Y_{3}\right|$ and taking $M_{S}$ as the mass of $S^{\prime}(\mathbf{1}, \mathbf{2})_{+1 / 2}$. From Eq. (22) one can estimate that for $M_{\psi} \gtrsim M_{S} \simeq 1 \mathrm{TeV}$ decay lengths will become larger than the order of millimeter for Yukawas smaller than $10^{-3}$. For Yukawas as small as $10^{-6}$, see Eq. (12), lifetimes exceed already $10 \mathrm{~s}$. Thus, the $\psi$ will hadronize before decaying. ATLAS studied constraints on long-lived colored particles [109], again in the context of a supersymmetric model. From Figs. 9 and 11 in Ref. [109] one can estimate that $\psi$ should be heavier than $M_{\psi} \gtrsim$ $1.8-1.9 \mathrm{TeV}$ for $c \tau=3-10 \mathrm{~m}$. From Ref. [110] one can estimate that similar numbers will apply also for quasistable $\psi$.

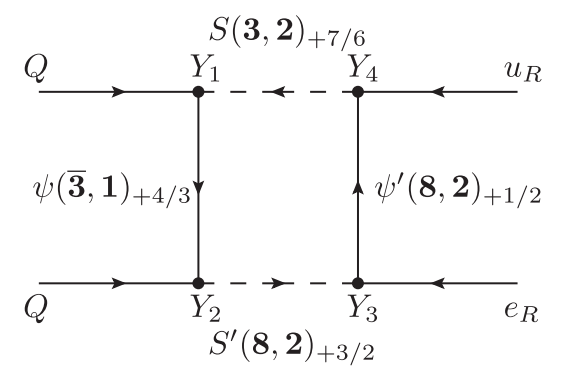

FIG. 4. An example, model II, for one-loop decomposition of the proton decay operator $\mathrm{O}_{2}$.

In summary, model I allows us to connect proton decay, dark matter, and neutrino masses. If the masses of the loop particles are of order of $\mathcal{O}(1-2) \mathrm{TeV}$, one can have also a wide range of interesting signals at the LHC. We have discussed a few possible search strategies for the LHC for the colored particles in this model.

\section{Model II}

Let us consider now a model without additional discrete symmetries, which we categorized into the second class in Sec. I. The full new particle content of the model is

$$
\begin{aligned}
\psi(\overline{\mathbf{3}}, \mathbf{1})_{+4 / 3} & =\psi_{+4 / 3}, \quad S(\mathbf{3}, \mathbf{2})_{+7 / 6}=\left(S_{+2 / 3}, S_{+5 / 3}\right), \\
\psi^{\prime}(\mathbf{8}, \mathbf{2})_{+1 / 2} & =\left(\psi_{0}^{\prime}, \psi_{+1}^{\prime}\right), \quad S^{\prime}(\mathbf{8}, \mathbf{2})_{+3 / 2}=\left(S_{+1}^{\prime}, S_{+2}^{\prime}\right),
\end{aligned}
$$

which corresponds to choosing the first row in Table III of the decomposition of the $\mathcal{O}_{2}$ operator with the parameter $\alpha=4 / 3$ for the electroweak $U(1)$ hypercharge. For the color representation, we take \#6 in Table I. The corresponding Feynman diagram of the one-loop proton decay is shown in Fig. 4.

The symmetries allow us to have an additional interaction

$$
\mathcal{L}_{2}=Y_{5} \overline{u_{R}} L \mathrm{i} \tau^{2} S+\text { H.c., }
$$

which does not appear in the one-loop proton decay diagram, shown in Fig. 4. With this interaction, a $d=12$ effective operator $Q Q u_{R} e_{R} \overline{u_{R}} L \bar{L} u_{R}$ appears at tree level, as shown in Fig. 5, which causes five-body proton decays such us $p \rightarrow e^{+} e^{-} e^{+} \pi^{+} \pi^{-}$. However, the decay modes induced from the $d=12$ operator are subdominant; using Eq. (1), for $Y_{5} \sim 10^{-2}$, we can roughly estimate the contribution of these modes to the proton total decay width to be around 40 orders of magnitude smaller than the twobody proton decay induced by the $d=6$ effective operator $\mathcal{O}_{2}$ given through the one-loop diagram in Fig. 4.

Combining the information listed in Tables I and III, one can find the coefficient of the effective interaction $\mathcal{O}_{2}$ of proton decay as 


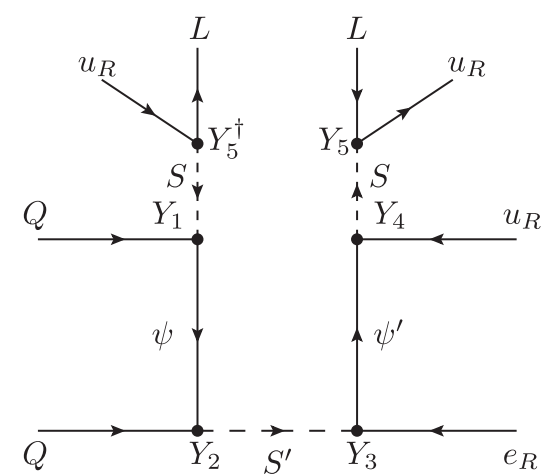

FIG. 5. $\quad d=12$ effective operator $Q Q u e \bar{u} L \bar{L} u$ at tree level.

$$
\mathcal{C}_{2}=-\frac{8}{3} M_{\psi} M_{\psi^{\prime}} I_{4} Y_{1} Y_{2} Y_{3} Y_{4}
$$

and the effective interaction causes only $p \rightarrow \pi^{0} e^{+}$. As we have seen in Sec. III A, the experimental bounds on the proton decay rate require Yukawa couplings of order $Y<$ $\mathcal{O}\left(10^{-6}\right)$ for the masses of the mediators at the $\mathrm{TeV}$ scale.

Within this model it is possible to have signatures that violate the lepton number by 1 unit, $\Delta L=1$. This can be seen in the pair production of the scalar $S_{+2}^{\prime}$ which has two possible decay modes $S_{+2}^{\prime} \rightarrow l^{+} l^{+} 2 j$ and $S_{+2}^{\prime} \rightarrow l^{+} 3 j$, as shown in Fig. 6. Therefore the pair production of the coloroctet $S_{+2}^{\prime}$ at the LHC might lead to the lepton number violating (LNV) signal 3 lepton plus 5 jets $\left(l^{+} l^{-} l^{ \pm} 5 j\right)$. Observation of LNV through this process is only possible if $\Gamma\left(S_{+2}^{\prime} \rightarrow l^{+} l^{+} 2 j\right)$ is of similar order to $\Gamma\left(S_{+2}^{\prime} \rightarrow l^{+} 3 j\right)$, since both final states are needed to establish that LNV is indeed taking place. Similar order of these decay widths are possible if $Y_{1} Y_{2} \sim Y_{3} Y_{4}$. Observing this LNV process also requires one to have short enough decays so the decays of the particles can be prompt.

This can be achieved for instance if we assume a mass hierarchy of the particles, $M_{S^{\prime}} \gtrsim M_{\psi}\left(M_{\psi^{\prime}}\right) \gtrsim M_{S}$, such that the pair production of $S_{+2}^{\prime}$ leads to a decay chain of twobody decays, as shown in Fig. 6. If this is the case, the

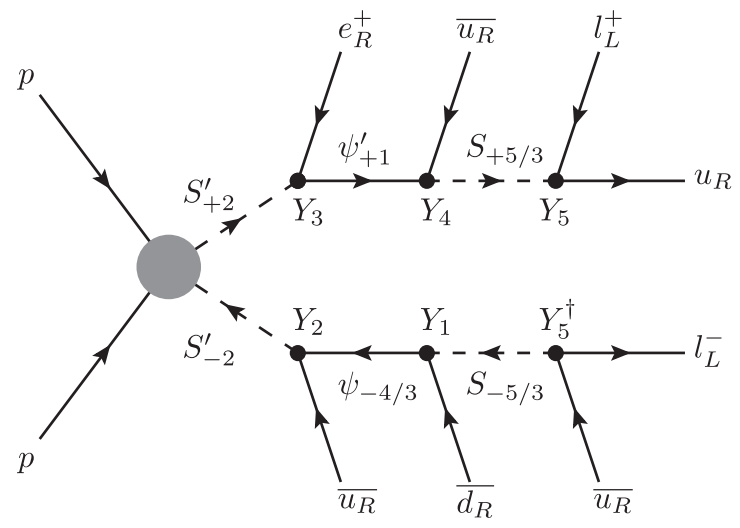

FIG. 6. Pair production and corresponding possible decays modes of the color-octet scalar $S_{+2}^{\prime}$ at the LHC.

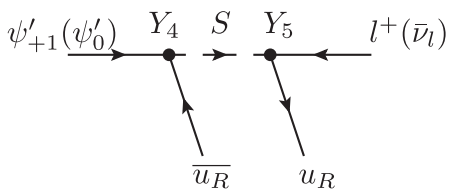

FIG. 7. Decay channels of the color-octet fermion $\psi^{\prime}$.

two decay modes of $S_{+2}^{\prime}$ will be $S_{+2}^{\prime} \rightarrow \psi_{+1}^{\prime} l^{+}$and $S_{+2}^{\prime} \rightarrow \psi_{+4 / 3} j$, and its decay length can be estimated as

$$
L_{0}\left(S_{+2}^{\prime}\right) \sim 10^{-2}[\mathrm{~m}] \frac{\left[10^{-6}\right]^{2}}{\left|Y_{3}\right|^{2}+\left|Y_{4}\right|^{2}}\left[\frac{\mathrm{TeV}}{M_{S^{\prime}}}\right] .
$$

Here, the choice for the Yukawa couplings $Y_{3}$ and $Y_{4}$ being order $10^{-6}$ is motivated by the current proton decay experimental bounds.

It is also possible to have long-lived particles at the LHC which are pair produced. Let us assume for instance that the color-octet fermion $\psi^{\prime}$ is slightly lighter than the scalar $S$. Then, the decay rates of the particles $\psi_{+1}^{\prime} \rightarrow l^{+} j j$ and $\psi_{0}^{\prime} \rightarrow$ $\nu_{l j} j$ can be estimated as (see Fig. 7)

$$
\Gamma\left(\psi^{\prime} \rightarrow l(\nu) j j\right) \sim \frac{\left|Y_{4} Y_{5}\right|^{2}}{512 \pi^{3}}\left[\frac{M_{\psi^{\prime}}}{M_{S}}\right]^{4} M_{\psi^{\prime}},
$$

which leads to the estimate of the decay length

$$
L_{0}\left(\psi^{\prime}\right) \sim 30[\mathrm{~m}]\left[\frac{10^{-6}}{\left|Y_{4}\right|}\right]^{2}\left[\frac{10^{-2}}{\left|Y_{5}\right|}\right]^{2}\left[\frac{\mathrm{TeV}}{M_{\psi}}\right]\left[\frac{M_{S}}{M_{\psi^{\prime}}}\right]^{4} .
$$

Here, the Yukawa coupling $Y_{5}$, which is not constrained by the proton decay, has been set to be of the order $10^{-2}$. Equation (28) shows that $\psi^{\prime}$, after being pair produced at the LHC, will become a long-lived particle. Since $\psi^{\prime}$ has also color, one can use $R$ hadron searches at the LHC [109,111-115] to constrain it.

In summary, in model II, depending on the mass hierarchies of the particles that appear in the proton decay diagram, one can have either long-lived colored and charged particles or prompt decays. The latter would allow us to establish experimentally the existence of LNV.

\section{CONCLUSIONS}

We have studied systematically the one-loop decomposition of the $d=6 B+L$ violating operators involving only SM fields. Our results are listed in tabular forms, from which all possible one-loop ultraviolet completions of these operators involving fermions and scalars can be constructed. We have briefly discussed how to use the information provided to calculate all coefficients that enter the calculation of the proton decay rate.

We then discussed that all models, in general, can be divided into two distinct classes. Class-I models are those for which the one-loop proton decay exists, but is not necessarily the dominant diagram for a given model. 
Models in this class therefore need usually an additional symmetry, such that tree-level contributions to proton decay become forbidden. Class-II models are then simply those for which the particle content guarantees that the oneloop diagram is automatically the dominant contribution to proton decay.

We then turned to possible phenomenology of these models and discussed one example model from each class. Class-I models have an absolutely stable particle and thus proton decay can be connected to the dark matter in the Universe. In the example we discussed, the same symmetry is responsible for one-loop proton decay, dark matter, and neutrino mass. The latter is also generated at one-loop level, as in the scotogenic neutrino mass model. We have discussed existing constraints and possible LHC phenomenology of this model also briefly.

For the example model of class II, we have discussed possible LHC phenomenology. It has been shown that depending on the mass hierarchy of the particles in the model, we can have particles that after being pair produced at the LHC can decay promptly, leading to LNV signals, or are long-lived colored and charged particles. Signals without missing energy, such as the LNV signals discussed, do not appear in model I and so can be used to distinguish between these two classes of models.

\section{ACKNOWLEDGMENTS}

M. H. was funded by Spanish Grants No. FPA201790566-REDC (Red Consolider MultiDark), No. FPA201785216-P, and No. SEV-2014-0398 (MINECO/AEI/FEDER, UE), as well as PROMETEO/2018/165 (Generalitat Valenciana). This work is supported in part by European Union's Horizon 2020 Research and Innovation Programme under the 777419-ESSnuSB, as well as by the COST Action CA15139 EuroNuNet. T. O. acknowledges the support of "Spanish Agencia Estatal de Investigación" (AEI) through the Grant "IFT Centro de Excelencia Severo Ochoa SEV2016-0597" and the EU "Fondo Europeo de Desarrollo Regional" (FEDER) through the project FPA2016-78645-P. J. C. H. is supported by Chile Grant Fondecyt No. 1161463.

\section{APPENDIX: FACTORS IN TABLES}

Here we write down the basis operators given in Eqs. (2)-(6) with all the indices of the SM gauge groups and Lorentz spinors,

$$
\begin{aligned}
\mathcal{O}_{1} & \equiv \epsilon^{I J K}\left[\left(\overline{d_{R}}\right)_{I \dot{a}}\left(u_{R}\right)_{J}^{\dot{a}}\right]\left[\left(\overline{Q^{c}}\right)_{K i}^{a}\left(\mathrm{i} \tau^{2}\right)^{i j}(L)_{j a}\right], \\
\mathcal{O}_{2} & \equiv \epsilon^{I J K}\left[\left(\overline{Q^{c}}\right)_{I i}^{a}\left(\mathrm{i} \tau^{2}\right)^{i j}(Q)_{J j a}\right]\left[\left(\overline{u_{R}^{c}}\right)_{K \dot{a}}\left(e_{R}\right)^{\dot{a}}\right], \\
\mathcal{O}_{3} & \equiv \epsilon^{I J K}\left[\left(\overline{Q^{c}}\right)_{I i}^{a}\left(\mathrm{i} \tau^{2}\right)^{i j}(Q)_{J j a}\right]\left[\left(\overline{Q^{c}}\right)_{K k}^{b}\left(\mathrm{i} \tau^{2}\right)^{k l}(L)_{l b}\right], \\
\mathcal{O}_{4} & \equiv \epsilon^{I J K}\left[\left(\overline{Q^{c}}\right)_{I i}^{a}\left(\mathrm{i} \tau^{2} \tau^{d}\right)^{i j}(Q)_{J j a}\right]\left[\left(\overline{Q^{c}}\right)_{K k}^{b}\left(\mathrm{i} \tau^{2} \tau^{d}\right)^{k l}(L)_{l b}\right], \\
\mathcal{O}_{5} & \equiv \epsilon^{I J K}\left[\left(\overline{{\overline{d_{R}}}^{c}}\right)_{I \dot{a}}\left(u_{R}\right)_{J}^{\dot{a}}\right]\left[\left(\overline{u_{R}}\right)_{K \dot{b}}\left(e_{R}\right)^{\dot{b}}\right],
\end{aligned}
$$

where the different indices are introduced to describe the different representations: $I, J, K \in\{1,2,3\}$ for a triplet under the color $S U(3), i, j, k, l \in\{1,2\}$ for a doublet under the electroweak $S U(2), d \in\{1,2,3\}$ on the Pauli matrices $\tau^{d}$ for a triplet under the $S U(2), a, b \in\{1,2\}$ for a lefthanded 2-spinor and $\dot{a}, \dot{b} \in\{\dot{1}, \dot{2}\}$ for a right-handed 2 -spinor. The position of the indices also depends on the representation of the field: a lower $I$ for $\mathbf{3}_{I}$ and an upper $I$ for $\overline{\mathbf{3}}^{I}$. The index on the $\overline{\mathbf{2}}^{i}$ representation of $S U(2)$ can be lowered as $\boldsymbol{2}_{i}$ with $\left(\mathrm{i} \tau^{2}\right)_{i j}$. On the position of the spinor indices, we follow the notation that is widely adopted in literature, e.g., Ref. [116]; the standard positions are determined as $\left(\psi_{L}\right)_{a}$ and $\left(\psi_{R}\right)^{\dot{a}}$ and the contraction is taken as $\left(\overline{\psi_{R}}\right)^{a}\left(\psi_{L}\right)_{a}$ and $\left(\overline{\psi_{L}}\right)_{\dot{a}}\left(\psi_{R}\right)^{\dot{a}}$ to form Lorentz scalars.

The ordering of the field operators in the decomposed interactions are determined as given in Eq. (7). In order to make the Yukawa interactions singlets under the color $S U(3)$, we plug the total antisymmetric tensors ( $\epsilon^{I J K}$ and $\left.\epsilon_{I J K}\right)$, the Gell-Mann matrices $\left[\left(\lambda^{A}\right)_{I}^{J}\right]$, and the ClebschGordan (CG) coefficient matrices $\left[\left(T_{\mathbf{6}}\right)_{X}^{I J}\right.$ and $\left.\left(T_{\overline{\mathbf{6}}}\right)_{I J}^{X}\right]$ into the interactions accordingly, where the index $A \in\{1 \cdots 8\}$ is for an octet, and a lower (upper) $X \in\{1 \cdots 6\}$ is for $\mathbf{6}$ $(\overline{\mathbf{6}})$. For the contraction of the electroweak $S U(2)$ indices, we use the antisymmetric tensors $\left[\left(\mathrm{i} \tau^{2}\right)^{i j}\right.$ and $\left.\left(\mathrm{i} \tau^{2}\right)_{i j}\right]$ and the Pauli matrices $\left[\left(\tau^{d}\right)_{i}{ }^{j}\right]$. The CG matrices for the sextet representations are defined as

$$
\begin{aligned}
& \left(T_{\mathbf{6}}\right)_{1}^{I J}=\left(\begin{array}{lll}
1 & & \\
& 0 & \\
& & 0
\end{array}\right), \quad\left(T_{\mathbf{6}}\right)_{2}^{I J}=\left(\begin{array}{ccc}
0 & \frac{1}{\sqrt{2}} & \\
\frac{1}{\sqrt{2}} & 0 & \\
& & 0
\end{array}\right), \\
& \left(T_{\mathbf{6}}\right)_{3}^{I J}=\left(\begin{array}{lll}
0 & & \\
& 1 & \\
& & 0
\end{array}\right), \quad\left(T_{\mathbf{6}}\right)_{4}^{I J}=\left(\begin{array}{ccc}
0 & \frac{1}{\sqrt{2}} \\
& 0 & \\
\frac{1}{\sqrt{2}} & & 0
\end{array}\right), \\
& \left(T_{\mathbf{6}}\right)_{5}^{I J}=\left(\begin{array}{lll}
0 & & \\
& 0 & \frac{1}{\sqrt{2}} \\
& \frac{1}{\sqrt{2}} & 0
\end{array}\right), \quad\left(T_{\mathbf{6}}\right)_{6}^{I J}=\left(\begin{array}{ccc}
0 & \\
& 0 & \\
& & 1
\end{array}\right),
\end{aligned}
$$

and $T_{\overline{6}}$ are defined in the same manner.

Let us demonstrate the operator projection (= reintegrate out the mediator fields), keeping all the indices, i.e., we explicitly derive a basis operator(s) from a decomposition with all the coefficients, signs, and factors. As an example, we take the basis operator $\mathcal{O}_{1}$ and decompose it with the mediators with \#4 in Table I, \#12 for Table II, i.e.,

$$
\begin{array}{cc}
\psi(\mathbf{3}, \mathbf{3})_{\alpha}, & S(\mathbf{8}, \mathbf{3})_{\alpha+1 / 3}, \\
\psi^{\prime}(\mathbf{6}, \mathbf{3})_{\alpha-1 / 3} & S^{\prime}(\mathbf{6}, \mathbf{2})_{\alpha+1 / 6} .
\end{array}
$$

The Yukawa interactions are defined as 


$$
\begin{aligned}
\mathcal{L}= & Y_{1}\left(\overline{{d_{R}}^{c}}\right)_{I \dot{a}}\left(\lambda^{\top^{A}}\right)_{I^{\prime}}{ }\left(\psi_{L}^{c}\right)^{I^{\prime} d \dot{a}} S^{A d}+Y_{2}\left(\overline{\psi_{L}^{c}}\right)_{J^{\prime}}^{d^{\prime} a}\left(T_{\mathbf{6}}\right)_{X}^{J^{\prime} J}(Q)_{J i a}\left(\tau^{T^{d^{\prime}}}\right)_{i^{\prime}}^{i}\left(S^{\prime \dagger}\right)^{X i^{\prime}}+Y_{3}\left(\overline{\psi_{R}^{\prime}}\right)^{X^{\prime} f b}(L)_{j b}\left(\mathrm{i} \tau^{2} \tau^{f}\right)^{j j^{\prime}} S_{X^{\prime} j^{\prime}}^{\prime} \\
& +Y_{4} \epsilon^{K L M}\left(\overline{u_{R}}\right)_{K \dot{b}}\left(\lambda^{A^{\prime}}\right)_{L}^{N}\left(T_{\overline{\mathbf{6}}}\right)_{N M}^{Y}\left(\psi_{R}^{\prime}\right)_{Y}^{f^{\prime} \dot{b}}\left(S^{\dagger}\right)^{A^{\prime} f^{\prime}}+\text { H.c. }
\end{aligned}
$$

The effective proton decay operator resulting from the box diagram mediated by them can be calculated as follows. First, the mediator fields are contracted, which give the propagators

$$
\begin{aligned}
& \mathcal{L}_{\text {eff }}=Y_{1} Y_{2} Y_{3} Y_{4}\left(\lambda^{\top A}\right)_{I^{\prime}}^{I}\left(T_{\mathbf{6}}\right)_{X}^{J^{\prime} J} \epsilon^{K L M}\left(\lambda^{A^{\prime}}\right)_{L}^{N}\left(T_{\overline{\mathbf{6}}}\right)_{N M}^{Y} \times\left(\tau^{\top d^{d^{\prime}}}\right)_{i^{\prime}}^{i}\left(\mathrm{i} \tau^{2} \tau^{f}\right)^{j j^{\prime}}\left\langle S^{A d}\left(S^{\dagger}\right)^{A^{\prime} f^{\prime}}\right\rangle\left\langle S_{X^{\prime} j^{\prime}}^{\prime}\left(S^{\prime \dagger}\right)^{X i^{\prime}}\right\rangle \\
& \times\left(\overline{d_{R}{ }^{c}}\right)_{I \dot{a}}\left\langle\left(\psi_{L}^{c}\right)^{I^{\prime} d \dot{a}}\left(\overline{\psi_{L}^{c}}\right)_{J^{\prime}}^{d^{\prime} a}\right\rangle(Q)_{J i a} \times\left(\overline{u_{R}^{c}}\right)_{K \dot{b}}\left\langle\left(\psi_{R}^{\prime}\right)_{Y}^{f^{\prime} \dot{b}}\left(\overline{\psi_{R}^{\prime}}\right)^{X^{\prime} f b}\right\rangle(L)_{j b} \\
& =Y_{1} Y_{2} Y_{3} Y_{4}\left(\lambda^{T^{A}}\right)^{I}{ }_{I^{\prime}}\left(T_{\mathbf{6}}\right)_{X}^{I^{\prime} J} \epsilon^{K L M}\left(\lambda^{A}\right)_{L}{ }^{N}\left(T_{\overline{\mathbf{6}}}\right)_{N M}^{X} \times\left(\mathrm{i} \tau^{2} \tau^{d}\right)^{j i^{\prime}}\left(\tau^{d}\right)_{i^{\prime}}{ }^{i} \int \frac{\mathrm{d}^{d} p}{(2 \pi)^{d}} \frac{\mathrm{i}}{p^{2}-M_{S}^{2}} \frac{\mathrm{i}}{p^{2}-M_{S^{\prime}}^{2}} \\
& \times\left(\overline{d_{R}^{c}}\right)_{I \dot{a}} \frac{-\mathrm{i} p_{\rho}\left(\bar{\sigma}^{\rho}\right)^{\dot{a} a}}{p^{2}-M_{\psi}^{2}}(Q)_{J i a}\left(\overline{u_{R}}\right)_{K \dot{b}} \frac{\mathrm{i} p_{\sigma}\left(\bar{\sigma}^{\sigma}\right)^{\dot{b} b}}{p^{2}-M_{\psi^{\prime}}^{2}}(L)_{j b}
\end{aligned}
$$

Next, the $S U(3)$ and the $S U(2)$ indices are rearranged,

$$
\mathcal{L}_{\text {eff }}=Y_{1} Y_{2} Y_{3} Y_{4}\left[4 \epsilon^{I J K}\right]\left[-3\left(\mathrm{i} \tau^{2}\right)^{i j}\right]\left[-\frac{1}{4} J_{4}\right] \times\left(\overline{d_{R}^{c}}\right)_{I \dot{a}}\left(\bar{\sigma}^{\rho}\right)^{\dot{a} a}(Q)_{J i a}\left(\overline{u_{R}^{c}}\right)_{K \dot{b}}\left(\bar{\sigma}_{\rho}\right)^{\dot{b} b}(L)_{j b} .
$$

Here we arrived at the step shown in Eq. (8). If necessary, the $S U(3)$ indices are renamed so that they fit to the ordering in the corresponding basis operator. This step may give an additional sign $[S U(3)$ sign in the tables],

$$
\mathcal{L}_{\text {eff }}=Y_{1} Y_{2} Y_{3} Y_{4}\left[4 \epsilon^{I J K}\right][-1]\left[-3\left(i \tau^{2}\right)^{i j}\right]\left[-\frac{1}{4} J_{4}\right] \times\left(\overline{d_{R}^{c}}\right)_{I \dot{a}}\left(\bar{\sigma}^{\rho}\right)^{\dot{a} a}(Q)_{K i a}\left(\overline{u_{R}^{c}}\right)_{J \dot{b}}\left(\bar{\sigma}_{\rho}\right)^{\dot{b} b}(L)_{j b}
$$

Finally, the Fierz transformation (rearrangement of the Lorentz indices) is carried out,

$$
\begin{aligned}
\mathcal{L}_{\text {eff }} & =Y_{1} Y_{2} Y_{3} Y_{4}[4][-1][-3]\left[-\frac{1}{4} J_{4}\right][2] \times \epsilon^{I J K}\left(\overline{d_{R}{ }^{c}}\right)_{I \dot{a}}\left(u_{R}\right)_{J}^{\dot{a}}\left(\overline{Q^{c}}\right)_{K i}^{a}\left(\mathrm{i} \tau^{2}\right)^{i j}(L)_{j a} \\
& =-6 Y_{1} Y_{2} Y_{3} Y_{4} J_{4} \mathcal{O}_{1} .
\end{aligned}
$$

In this example, we obtain 4 for the $S U(3)$ coefficient, - for the $S U(3)$ sign, -3 for the $S U(2)$ coefficient, 2 for the factor of the Fierz transformation for Lorentz indices, and $-J_{4} / 4$ for the loop integral factor. The loop integral factors $I_{4}$ and $J_{4}$ that appear in Tables II-V are defined as

$$
\begin{aligned}
I_{4} & \equiv \int \frac{\mathrm{d}^{4} k}{(2 \pi)^{4}} \mathrm{i} \frac{1}{\left(k^{2}-M_{\psi}^{2}\right)\left(k^{2}-M_{S}^{2}\right)\left(k^{2}-M_{\psi^{\prime}}^{2}\right)\left(k^{2}-M_{S^{\prime}}^{2}\right)}, \\
J_{4} & \equiv \int \frac{\mathrm{d}^{4} k}{(2 \pi)^{4}} \frac{k^{2}}{\left(k^{2}-M_{\psi}^{2}\right)\left(k^{2}-M_{S}^{2}\right)\left(k^{2}-M_{\psi^{\prime}}^{2}\right)\left(k^{2}-M_{S^{\prime}}^{2}\right)} .
\end{aligned}
$$

In the limit where all the mediator masses are identical, the integrals converge to

$$
I_{4} \rightarrow \frac{1}{16 \pi^{2}} \frac{1}{6} \frac{1}{M^{4}}, \quad J_{4} \rightarrow-\frac{1}{16 \pi^{2}} \frac{1}{3} \frac{1}{M^{2}},
$$

where $M$ is the common value of the masses. All the information to reproduce the coefficient of the effective operator $\mathcal{O}_{1}$ in Eq. (A8) can be found in Tables I and II. 
[1] S. Sussman et al. (Super-Kamiokande Collaboration), arXiv:1811.12430.

[2] K. Abe et al. (Super-Kamiokande Collaboration), Phys. Rev. D 96, 012003 (2017).

[3] K. Abe et al. (Super-Kamiokande Collaboration), Phys. Rev. D 95, 012004 (2017).

[4] V. Takhistov et al. (Super-Kamiokande Collaboration), Phys. Rev. Lett. 115, 121803 (2015).

[5] K. Asakura et al. (KamLAND Collaboration), Phys. Rev. D 92, 052006 (2015).

[6] J. Gustafson et al. (Super-Kamiokande Collaboration), Phys. Rev. D 91, 072009 (2015).

[7] V. Takhistov et al. (Super-Kamiokande Collaboration), Phys. Rev. Lett. 113, 101801 (2014).

[8] K. Abe et al. (Super-Kamiokande Collaboration), Phys. Rev. D 90, 072005 (2014).

[9] K. Abe et al. (Super-Kamiokande Collaboration), Phys. Rev. Lett. 113, 121802 (2014).

[10] M. Tanabashi et al. (Particle Data Group), Phys. Rev. D 98, 030001 (2018).

[11] K. Abe et al. (Hyper-Kamiokande Collaboration), arXiv: 1805.04163.

[12] B. Abi et al. (DUNE Collaboration), arXiv:1807.10334.

[13] F. An et al. (JUNO Collaboration), J. Phys. G 43, 030401 (2016).

[14] Y. Suzuki et al. (TITAND Working Group), in 11th International School on Particles and Cosmology Karbardino-Balkaria, Russia, 2001 (2001), pp. 288-296.

[15] M. D. Kistler, H. Yuksel, S. Ando, J. F. Beacom, and Y. Suzuki, Phys. Rev. D 83, 123008 (2011).

[16] A. de Bellefon et al., arXiv:hep-ex/0607026.

[17] S. Bser, M. Kowalski, L. Schulte, N. L. Strotjohann, and M. Voge, Astropart. Phys. 62, 54 (2015).

[18] D. Cowan, in Intensity Frontier Workshop, Argonne National Laboratory, 2013 (2013).

[19] H. Georgi and S. L. Glashow, Phys. Rev. Lett. 32, 438 (1974).

[20] P. Langacker, Phys. Rep. 72, 185 (1981).

[21] N. Sakai and T. Yanagida, Nucl. Phys. B197, 533 (1982).

[22] S. Weinberg, Phys. Rev. D 26, 287 (1982).

[23] S. Dimopoulos, S. Raby, and F. Wilczek, Phys. Lett. 112B, 133 (1982).

[24] J. R. Ellis, D. V. Nanopoulos, and S. Rudaz, Nucl. Phys. B202, 43 (1982).

[25] P. Nath, A. H. Chamseddine, and R. L. Arnowitt, Phys. Rev. D 32, 2348 (1985).

[26] J. Hisano, H. Murayama, and T. Yanagida, Nucl. Phys. B402, 46 (1993).

[27] V. Lucas and S. Raby, Phys. Rev. D 55, 6986 (1997).

[28] T. Goto and T. Nihei, Phys. Rev. D 59, 115009 (1999).

[29] H. Murayama and A. Pierce, Phys. Rev. D 65, 055009 (2002).

[30] J. Hisano, D. Kobayashi, T. Kuwahara, and N. Nagata, J. High Energy Phys. 07 (2013) 038.

[31] J. Ellis, J. L. Evans, F. Luo, N. Nagata, K. A. Olive, and P. Sandick, Eur. Phys. J. C 76, 8 (2016).

[32] K. S. Babu et al., in Proceedings, 2013 Community Summer Study on the Future of U.S. Particle Physics: Snowmass on the Mississippi (CSS2013), Minneapolis, MN, USA, 2013 (2013).
[33] S. Weinberg, Phys. Rev. Lett. 43, 1566 (1979).

[34] F. Wilczek and A. Zee, Phys. Rev. Lett. 43, 1571 (1979).

[35] L. F. Abbott and M. B. Wise, Phys. Rev. D 22, 2208 (1980).

[36] R. M. Fonseca, M. Hirsch, and R. Srivastava, Phys. Rev. D 97, 075026 (2018).

[37] K. S. Babu and R. N. Mohapatra, Phys. Rev. Lett. 109, 091803 (2012).

[38] L. Lehman, Phys. Rev. D 90, 125023 (2014).

[39] S. Bhattacharya and J. Wudka, Phys. Rev. D 94, 055022 (2016); 95, 039904(E) (2017).

[40] P. J. O'Donnell and U. Sarkar, Phys. Lett. B 316, 121 (1993).

[41] T. Hambye and J. Heeck, Phys. Rev. Lett. 120, 171801 (2018).

[42] R. M. Fonseca, J. Phys. Conf. Ser. 873, 012045 (2017).

[43] A. de Gouvea, J. Herrero-Garcia, and A. Kobach, Phys. Rev. D 90, 016011 (2014).

[44] E. Ma, Phys. Rev. D 73, 077301 (2006).

[45] R. Alonso, H.-M. Chang, E. E. Jenkins, A. V. Manohar, and B. Shotwell, Phys. Lett. B 734, 302 (2014).

[46] J. C. Helo, M. Hirsch, and T. Ota, J. High Energy Phys. 06 (2018) 047.

[47] F. Bonnet, M. Hirsch, T. Ota, and W. Winter, J. High Energy Phys. 07 (2012) 153.

[48] J.-S. Yoo, Y. Aoki, T. Izubuchi, and S. Syritsyn, arXiv:1812.09326.

[49] Y. Aoki, T. Izubuchi, E. Shintani, and A. Soni, Phys. Rev. D 96, 014506 (2017).

[50] Y. Aoki, E. Shintani, and A. Soni, Phys. Rev. D 89, 014505 (2014).

[51] Y. Aoki, C. Dawson, J. Noaki, and A. Soni, Phys. Rev. D 75, 014507 (2007).

[52] M. Claudson, M. B. Wise, and L. J. Hall, Nucl. Phys. B195, 297 (1982).

[53] S. Chadha and M. Daniel, Nucl. Phys. B229, 105 (1983).

[54] Y. Aoki, P. Boyle, P. Cooney, L. Del Debbio, R. Kenway, C. M. Maynard, A. Soni, and R. Tweedie (RBC-UKQCD Collaboration), Phys. Rev. D 78, 054505 (2008).

[55] J. C. Helo, M. Hirsch, and S. Kovalenko, Phys. Rev. D 89, 073005 (2014); 93, 099902(E) (2016).

[56] A. de la Puente and A. Szynkman, Eur. Phys. J. C 76, 124 (2016).

[57] J. Liu, Z. Liu, and L.-T. Wang, Phys. Rev. Lett. 122, 131801 (2019).

[58] L. Lee, C. Ohm, A. Soffer, and T.-T. Yu, Prog. Part. Nucl. Phys. 106, 210 (2019).

[59] G. Blanger et al., J. High Energy Phys. 02 (2019) 186.

[60] G. Cottin, J. C. Helo, and M. Hirsch, Phys. Rev. D 97, 055025 (2018).

[61] G. Cottin, J. C. Helo, and M. Hirsch, Phys. Rev. D 98, 035012 (2018).

[62] G. Cottin, J. C. Helo, M. Hirsch, and D. Silva, arXiv:1902.05673.

[63] J. P. Chou, D. Curtin, and H. J. Lubatti, Phys. Lett. B 767, 29 (2017).

[64] V. V. Gligorov, S. Knapen, B. Nachman, M. Papucci, and D. J. Robinson, Phys. Rev. D 99, 015023 (2019).

[65] D. Dercks, H. K. Dreiner, M. Hirsch, and Z. S. Wang, Phys. Rev. D 99, 055020 (2019). 
[66] J. C. Helo, M. Hirsch, and Z. S. Wang, J. High Energy Phys. 07 (2018) 056.

[67] D. Curtin et al., arXiv:1806.07396.

[68] A. M. Sirunyan et al. (CMS Collaboration), Phys. Rev. D 99, 032011 (2019).

[69] M. Aaboud et al. (ATLAS Collaboration), Phys. Rev. D 99, 052005 (2019).

[70] A. M. Sirunyan et al. (CMS Collaboration), J. High Energy Phys. 02 (2019) 179.

[71] M. Aaboud et al. (ATLAS Collaboration), Phys. Lett. B 788, 96 (2019).

[72] M. Aaboud et al. (ATLAS Collaboration), Phys. Rev. D 99, 012001 (2019).

[73] A. M. Sirunyan et al. (CMS Collaboration), Phys. Rev. D 98, 092011 (2018).

[74] A. M. Sirunyan et al. (CMS Collaboration), J. High Energy Phys. 08 (2018) 016.

[75] A. M. Sirunyan et al. (CMS Collaboration), J. High Energy Phys. 05 (2018) 025.

[76] A. M. Sirunyan et al. (CMS Collaboration), J. High Energy Phys. 05 (2018) 127.

[77] M. Aaboud et al. (ATLAS Collaboration), J. High Energy Phys. 06 (2018) 022.

[78] A. M. Sirunyan et al. (CMS Collaboration), Phys. Lett. B 780, 432 (2018).

[79] M. Aaboud et al. (ATLAS Collaboration), Phys. Rev. D 97, 052012 (2018).

[80] V. Khachatryan et al. (CMS Collaboration), Phys. Rev. D 95, 012009 (2017).

[81] M. Aaboud et al. (ATLAS Collaboration), Phys. Rev. D 93, 112015 (2016).

[82] G. Aad et al. (ATLAS Collaboration), Phys. Rev. D 92, 072004 (2015).

[83] V. Khachatryan et al. (CMS Collaboration), Phys. Rev. D 91, 052012 (2015).

[84] V. Khachatryan et al. (CMS Collaboration), Phys. Rev. D 91, 012007 (2015).

[85] S. Chatrchyan et al. (CMS Collaboration), J. High Energy Phys. 07 (2013) 122.

[86] J. Kubo, E. Ma, and D. Suematsu, Phys. Lett. B 642, 18 (2006).

[87] C. Boehm, Y. Farzan, T. Hambye, S. Palomares-Ruiz, and S. Pascoli, Phys. Rev. D 77, 043516 (2008).

[88] D. Aristizabal Sierra, J. Kubo, D. Restrepo, D. Suematsu, and O. Zapata, Phys. Rev. D 79, 013011 (2009).

[89] D. Suematsu, T. Toma, and T. Yoshida, Phys. Rev. D 79, 093004 (2009).

[90] G. B. Gelmini, E. Osoba, and S. Palomares-Ruiz, Phys. Rev. D 81, 063529 (2010).
[91] D. Schmidt, T. Schwetz, and T. Toma, Phys. Rev. D 85, 073009 (2012).

[92] D. Restrepo, O. Zapata, and C. E. Yaguna, J. High Energy Phys. 11 (2013) 011.

[93] J. Racker, J. Cosmol. Astropart. Phys. 03 (2014) 025.

[94] T. Toma and A. Vicente, J. High Energy Phys. 01 (2014) 160.

[95] E. Molinaro, C. E. Yaguna, and O. Zapata, J. Cosmol. Astropart. Phys. 07 (2014) 015.

[96] A. Vicente and C. E. Yaguna, J. High Energy Phys. 02 (2015) 144.

[97] A. G. Hessler, A. Ibarra, E. Molinaro, and S. Vogl, J. High Energy Phys. 01 (2017) 100.

[98] C. Hagedorn, J. Herrero-Garca, E. Molinaro, and M. A. Schmidt, J. High Energy Phys. 11 (2018) 103.

[99] L. Lavoura, Eur. Phys. J. C 29, 191 (2003).

[100] A. M. Baldini et al. (MEG Collaboration), Eur. Phys. J. C 76, 434 (2016).

[101] L. Calibbi and G. Signorelli, Riv. Nuovo Cimento 41, 71 (2018).

[102] L. Lopez Honorez, E. Nezri, J. F. Oliver, and M. H. G. Tytgat, J. Cosmol. Astropart. Phys. 02 (2007) 028.

[103] A. Arhrib, Y.-L. S. Tsai, Q. Yuan, and T.-C. Yuan, J. Cosmol. Astropart. Phys. 06 (2014) 030.

[104] G. Sigl, Astroparticle Physics: Theory and Phenomenology, Atlantis Studies in Astroparticle Physics and Cosmology Vol. 1 (Springer, Hamburg, Germany, 2017).

[105] A. M. Sirunyan et al. (CMS Collaboration), Phys. Rev. D 96, 032003 (2017).

[106] M. Aaboud et al. (ATLAS Collaboration), Phys. Rev. D 97, 112001 (2018).

[107] S. Beauceron (CMS Collaboration), Technical Report CMS-CR-2018-378, CERN, Geneva (2018).

[108] M. Aaboud et al. (ATLAS Collaboration), New J. Phys. 18, 093016 (2016).

[109] M. Aaboud et al. (ATLAS Collaboration) arXiv:1902. 01636.

[110] J. Heinrich, Ph.D. thesis, Munich University.

[111] J. Alimena et al., arXiv:1903.04497.

[112] G. R. Farrar, R. Mackeprang, D. Milstead, and J. P. Roberts, J. High Energy Phys. 02 (2011) 018.

[113] F. Buccella, G. R. Farrar, and A. Pugliese, Phys. Lett. B 153, 311 (1985).

[114] CMS Collaboration, Report No. CMS-PAS-EXO-16-036, 2016.

[115] Z. Liu and B. Tweedie, J. High Energy Phys. 06 (2015) 042.

[116] H. E. Haber and G. L. Kane, Phys. Rep. 117, 75 (1985). 